\title{
WHAT IS BOOLEAN VALUED ANALYSIS?
}

\author{
S. S. KUTATELADZE
}

Abstract. This is a brief overview of the basic techniques of Boolean valued analysis.

\section{Introduction}

The term "Boolean valued analysis" appeared within the realm of mathematical logic. It was Takeuti, a renowned expert in proof theory, who introduced the term. Takeuti defined Boolean valued analysis in [1 p. 1] as "an application of ScottSolovay's Boolean valued models of set theory to analysis." Vopěnka invented similar models at the same time. That is how the question of the title receives an answer in zero approximation. However, it would be premature to finish at this stage. It stands to reason to discuss in more detail the following three questions.

1.1. Why should we know anything at all about Boolean valued analysis? Curiosity often leads us in science, and oftener we do what we can. However we appreciate that which makes us wiser. Boolean valued analysis has this value, expanding the limits of our knowledge and taking off blinds from the eyes of the perfect mathematician, mathematician par excellence. To substantiate this thesis is the main target of the exposition to follow.

1.2. What need the working mathematician know this for? Part of the answer was given above: to become wiser. There is another, possibly more important, circumstance. Boolean valued analysis not only is tied up with many topological and geometrical ideas but also provides the technology for expanding the content of the already available theorems. Every theorem, proven by the classical means, possesses some new and nonobvious content that relates to "variable sets." Speaking more strictly, each of the available theorems generates a whole family of its next of kin in disguise which is enumerated by all complete Boolean algebras or, equivalently, nonhomeomorphic Stone spaces.

1.3. What do the Boolean valued models yield? The essential and technical parts of this survey are devoted to answering the question. We will focus on the general methods independent of the subtle intrinsic properties of the initial complete Boolean algebra. These methods are simple, visual, and easy to apply. Therefore they may be useful for the working mathematician. Moreover, we must always keep in mind that the Boolean valued models were invented in order to simplify the exposition of Cohen's forcing [2]. Mathematics is impossible without proof. Nullius in Verbia. Therefore, part of exposition is allotted to the scheme of proving the consistency of the negation of the continuum hypothesis with the axioms of

Key words and phrases. Boolean valued model, ascent, descent, continuum hypothesis.

An expanded version of a talk at Taimanov's seminar on geometry, topology, and their applications in the Sobolev Institute of Mathematics on September 25, 2006. 
Zermelo-Fraenkel set theory (with choice) ZFC. Cohen was awarded a Fields medal in 1966 for this final step in settling Hilbert's problem No. $1 .^{1}$

\section{Boolean Valued Models}

The stage of Boolean valued analysis is some Boolean valued model of ZFC. To define these models, we start with a complete Boolean algebra $B .^{2}$

\subsection{A Boolean valued universe. Given an ordinal $\alpha$, put}

$$
\mathbb{V}_{\alpha}^{(B)}:=\left\{x \mid \operatorname{Fnc}(x) \wedge(\exists \beta)\left(\beta<\alpha \wedge \operatorname{dom}(x) \subset \mathbb{V}_{\beta}^{(B)} \wedge \operatorname{im}(x) \subset B\right)\right\} .
$$

(The relevant information on ordinals is collected below in 7.2.) In more detail,

$$
\begin{gathered}
\mathbb{V}_{0}^{(B)}:=\varnothing, \\
\mathbb{V}_{\alpha+1}^{(B)}:=\left\{x \mid x \text { is a function, } \operatorname{dom}(x) \subset \mathbb{V}_{\alpha}^{(B)}, \operatorname{im}(x) \subset B\right\}, \\
\mathbb{V}_{\alpha}^{(B)}:=\bigcup_{\beta<\alpha} \mathbb{V}_{\beta}^{(B)} \quad(\alpha \text { is a limit ordinal }) .
\end{gathered}
$$

The class

$$
\mathbb{V}^{(B)}:=\bigcup_{\alpha \in \mathrm{On}} \mathbb{V}_{\alpha}^{(B)}
$$

is a Boolean valued universe. The elements of $\mathbb{V}^{(B)}$ are $B$-valued sets. Note that $\mathbb{V}^{(B)}$ consists only of functions. In particular, $\varnothing$ is the function with domain $\varnothing$ and range $\varnothing$. Consequently, the three "lower" floors of $\mathbb{V}^{(B)}$ are composed as follows: $\mathbb{V}_{0}^{(B)}=\varnothing, \mathbb{V}_{1}^{(B)}=\{\varnothing\}$, and $\mathbb{V}_{2}^{(B)}=\{\varnothing,(\{\varnothing\}, b) \mid b \in B\}$. Observe also that $\alpha \leq \beta \rightarrow \mathbb{V}_{\alpha}^{(B)} \subset \mathbb{V}_{\beta}^{(B)}$ for all ordinals $\alpha$ and $\beta$. Moreover, $\mathbb{V}^{(B)}$ enjoys the induction principle

$$
\left(\forall x \in \mathbb{V}^{(B)}\right)((\forall y \in \operatorname{dom}(x)) \varphi(y) \rightarrow \varphi(x)) \rightarrow\left(\forall x \in \mathbb{V}^{(B)}\right) \varphi(x),
$$

with $\varphi$ a formula of ZFC.

2.2. Truth values. Consider a formula $\varphi$ of ZFC, where $\varphi=\varphi\left(u_{1}, \ldots, u_{n}\right)$. If we replace $u_{1}, \ldots, u_{n}$ with $x_{1}, \ldots, x_{n} \in \mathbb{V}^{(B)}$, then we obtain a particular assertion about the objects $x_{1}, \ldots, x_{n}$. We will try to ascribe to this assertion some truth value. This truth value $\llbracket \varphi \rrbracket$ must be a member of $B$. Moreover, we desire naturally that all theorems of ZFC become "as valid as possible" with respect to the new procedure, so that the top $\mathbb{1}:=\mathbb{1}_{B}$ of $B$, i.e. the unity of $B$, serves as the truth value of a theorem of ZFC.

The truth value of a well-formed formula must be determined by "double" recursion. On the one hand, we must induct on the length of a formula, considering the way it is constructed from the atomic formulas of the shape $x \in y$ and $x=y$. On the other hand, we must define the truth values of the atomic formulas while $x$ and $y$ range over $\mathbb{V}^{(B)}$, on using the recursive construction of the Boolean valued universe.

\footnotetext{
${ }^{1}$ Hilbert [3] considered it plausible that "as regards equivalence there are, therefore, only two assemblages of numbers, the countable assemblage and the continuum."

${ }^{2} \mathrm{~A}$ Boolean algebra is a unital algebra, over the two-point field, whose every element is idempotent. Given $a, b \in B$, put $a \leq b \leftrightarrow a b=a$. The completeness of $B$ means the existence of the least upper and greatest lower bounds of each subset of $B$.
} 
Clearly, if $\varphi$ and $\psi$ are already evaluated formulas of $\mathrm{ZFC}$, and $\llbracket \varphi \rrbracket \in B$ and $\llbracket \psi \rrbracket \in B$ are the truth values of thee formulas then we must put

$$
\begin{aligned}
\llbracket \varphi \wedge \psi \rrbracket & :=\llbracket \varphi \rrbracket \wedge \llbracket \psi \rrbracket, \\
\llbracket \varphi \vee \psi \rrbracket: & :=\llbracket \varphi \rrbracket \vee \llbracket \psi \rrbracket, \\
\llbracket \varphi \rightarrow \psi \rrbracket & :=\llbracket \varphi \rrbracket \rightarrow \llbracket \psi \rrbracket, \\
\llbracket \neg \varphi \rrbracket: & :=\neg \llbracket \varphi \rrbracket, \\
\llbracket(\forall x) \varphi(x) \rrbracket & :=\bigwedge_{x \in \mathbb{V}(B)} \llbracket \varphi(x) \rrbracket, \\
\llbracket(\exists x) \varphi(x) \rrbracket & :=\bigvee_{x \in \mathbb{V}(B)} \llbracket \varphi(x) \rrbracket,
\end{aligned}
$$

where the right-hand sides contain the Boolean operations that correspond to the logical connectives and quantifiers on the left-hand sides: $\wedge$ is the meet, $\vee$ is the join, $\neg$ is the complementation, and the implication $\rightarrow$ is defined by the rule $a \rightarrow$ $b:=\neg a \vee b$ for $a, b \in B$. Only these definitions ensure the value "unity" for the truth values of the classical tautologies.

We turn now to evaluating the atomic formulas $x \in y$ and $x=y$ for $x, y \in \mathbb{V}^{(B)}$. The intuitive idea behind the definition states that every $B$-valued set $y$ is "fuzzy," i.e. "it contains each member $z$ of $\operatorname{dom}(y)$ with probability $y(z)$." Bearing this in mind and attempting to preserve the logical tautology $x \in y \leftrightarrow(\exists z \in y)(x=z)$ alongside the axiom of extensionality, we arrive to the following recursive definition:

$$
\begin{gathered}
\llbracket x \in y \rrbracket:=\bigvee_{z \in \operatorname{dom}(y)} y(z) \wedge \llbracket z=x \rrbracket, \\
\llbracket x=y \rrbracket:=\bigwedge_{z \in \operatorname{dom}(x)} x(z) \rightarrow \llbracket z \in y \rrbracket \wedge \bigwedge_{z \in \operatorname{dom}(y)} y(z) \rightarrow \llbracket z \in x \rrbracket .
\end{gathered}
$$

We can now ascribe some meaning to the formal records like $\varphi\left(x_{1}, \ldots, x_{n}\right)$, where $x_{1}, \ldots, x_{n} \in \mathbb{V}^{(B)}$ and $\varphi$ is a formula of ZFC. In other words, we are able to define the strict sense in which the set theoretic expression $\varphi\left(u_{1}, \ldots, u_{n}\right)$ is valid for $x_{1}, \ldots, x_{n} \in \mathbb{V}^{(B)}$.

Namely, we will say that a formula $\varphi\left(x_{1}, \ldots, x_{n}\right)$ is satisfied inside $\mathbb{V}^{(B)}$ or the collection of $x_{1}, \ldots, x_{n}$ possesses the property $\varphi$ inside $\mathbb{V}^{(B)}$ provided that $\llbracket \varphi\left(x_{1}, \ldots, x_{n}\right) \rrbracket=\mathbb{1}$. In this event we write $\mathbb{V}^{(B)} \models \varphi\left(x_{1}, \ldots, x_{n}\right)$. If some formula $\varphi$ of ZFC is expressed in the natural language of discourse then by way of pedantry we use quotes: $\mathbb{V}^{(B)} \models " \varphi$." The satisfaction mark $\models$ implies the usage of the model-theoretic expressions of the kind " $\vee(B)$ is a Boolean valued model for $\varphi$ " instead of $\mathbb{V}^{(B)} \models \varphi$.

All axioms of the first-order predicate calculus are obviously valid inside $\mathbb{V}^{(B)}$. In particular,

(1) $\llbracket x=x \rrbracket=\mathbb{1}$,

(2) $\llbracket x=y \rrbracket=\llbracket y=x \rrbracket$,

(3) $\llbracket x=y \rrbracket \wedge \llbracket y=z \rrbracket \leq \llbracket x=z \rrbracket$,

(4) $\llbracket x=y \rrbracket \wedge \llbracket z \in x \rrbracket \leq \llbracket z \in y \rrbracket$,

(5) $\llbracket x=y \rrbracket \wedge \llbracket x \in z \rrbracket \leq \llbracket y \in z \rrbracket$.

Observe that $\mathbb{V}^{(B)} \models x=y \wedge \varphi(x) \rightarrow \varphi(y)$ for every formula $\varphi$, i.e.

(6) $\llbracket x=y \rrbracket \wedge \llbracket \varphi(x) \rrbracket \leq \llbracket \varphi(y) \rrbracket$. 


\section{Principles of Boolean Valued Analysis}

The equality $\llbracket x=y \rrbracket=\mathbb{1}$ holding in the Boolean valued universe $\mathbb{V}^{(B)}$ implies in no way that the functions $x$ and $y$ coincide in their capacities of members of $\mathbb{V}$. For instance, the function vanishing at one of the levels $\mathbb{V}_{\alpha}^{(B)}$, with $\alpha \geq 1$, plays the role of the empty set inside $\mathbb{V}^{(B)}$. This circumstance is annoying in a few constructions we use in the sequel.

3.1. The separated universe. To overcome this nuisance, we will pass from $\mathbb{V}^{(B)}$ to the separated Boolean valued universe $\overline{\mathbb{V}}^{(B)}$, keeping for the latter the previous symbol $\mathbb{V}^{(B)}$; i.e. we put $\mathbb{V}^{(B)}:=\overline{\mathbb{V}}^{(B)}$. Furthermore, to define $\overline{\mathbb{V}}^{(B)}$, we consider the equivalence $\{(x, y) \mid \llbracket x=y \rrbracket=\mathbb{1}\}$ on $\mathbb{V}^{(B)}$. Choosing a member (representative of the least rank) in each class of equivalent functions, we come in fact to the separated universe $\overline{\mathbb{V}}^{(B)}$.

Note that the equality

$$
\llbracket x=y \rrbracket=\mathbb{1} \rightarrow \llbracket \varphi(x) \rrbracket=\llbracket \varphi(y) \rrbracket
$$

holds for every formula $\varphi$ of ZFC and members $x, y$ of $\mathbb{V}^{(B)}$. Therefore, we may evaluate formulas over the separated universe, disregarding the choice of representatives. Dealing with the separated universe in the sequel, we will consider, for the sake of convenience, a particular representative of a class of equivalence rather than the whole class itself (in contrast to the conventional practice of analysis in treating the function spaces of the Riesz scale).

3.2. Properties of a Boolean valued model. The main properties of a Boolean valued universe $\mathbb{V}^{(B)}$ are collected in the following three propositions:

(1) Transfer Principle. All theorems of ZFC are valid inside $\mathbb{V}^{(B)}$; i.e., in symbols,

$$
\mathbb{V}^{(B)} \models \mathrm{ZFC} .
$$

The transfer principle is established by a bulky check that the truth value of every axiom of $\mathrm{ZFC}$ is $\mathbb{1}$ and all inference rules increase truth values. The transfer principle reads sometimes as follows: " $\mathbb{V}^{(B)}$ is a Boolean valued model of ZFC." That is how the term "Boolean valued model of set theory" enters the realms of mathematics.

(2) Maximum Principle. To each formula $\varphi$ of ZFC there is a member $x_{0}$ of $\mathbb{V}^{(B)}$ satisfying $\llbracket(\exists x) \varphi(x) \rrbracket=\llbracket \varphi\left(x_{0}\right) \rrbracket$.

In particular, it is true inside $\mathbb{V}^{(B)}$ that if $\varphi(x)$ for some $x$ then there exists a member $x_{0}$ of $\mathbb{V}^{(B)}$ (in the sense of $\mathbb{V}$ ) satisfying $\llbracket \varphi\left(x_{0}\right) \rrbracket=\mathbb{1}$. In symbols,

$$
\mathbb{V}^{(B)} \models(\exists x) \varphi(x) \rightarrow\left(\exists x_{0}\right) \mathbb{V}^{(B)} \models \varphi\left(x_{0}\right) .
$$

The maximum principle means that $\left(\exists x_{0} \in \mathbb{V}^{(B)}\right) \llbracket \varphi\left(x_{0}\right) \rrbracket=\bigvee_{x \in \mathbb{V}(B)} \llbracket \varphi(x) \rrbracket$ for every formula $\varphi$ of ZFC. The last formula illuminates the background behind the term "maximum principle." The proof of the principle consists in an easy application of the following fact:

(3) Mixing Principle. Let $\left(b_{\xi}\right)_{\xi \in \Xi}$ be a partition of unity in $B$; i.e., a family of members of $B$ such that

$$
\bigvee_{\xi \in \Xi} b_{\xi}=\mathbb{1}, \quad(\forall \xi, \eta \in \Xi)\left(\xi \neq \eta \rightarrow b_{\xi} \wedge b_{\eta}=\mathbb{0}\right)
$$


For every family $\left(x_{\xi}\right)_{\xi \in \Xi}$ of elements of $\mathbb{V}^{(B)}$ and every partition of unity $\left(b_{\xi}\right)_{\xi \in \Xi}$ there is a unique mixing $\left(x_{\xi}\right)$ by $\left(b_{\xi}\right)$ (or with probabilities $\left(b_{\xi}\right)$ ); i.e., a member $x$ of the separated universe $\mathbb{V}^{(B)}$ satisfying $b_{\xi} \leq \llbracket x=x_{\xi} \rrbracket$ for all $\xi \in \Xi$. The mixing $x$ of a family $\left(x_{\xi}\right)$ by $\left(b_{\xi}\right)$ is denoted as follows: $x=\operatorname{mix}_{\xi \in \Xi}\left(b_{\xi} x_{\xi}\right)=\operatorname{mix}\left\{b_{\xi} x_{\xi} \mid \xi \in \Xi\right\}$. Mixing is connected with the main particularity of a Boolean valued model, the procedure of collecting the highest truth value "stepwise."

(4) Take $x \in \mathbb{V}^{(B)}$ and $b \in B$. Define the function $b x$ on $\operatorname{dom}(b x):=\operatorname{dom}(x)$ by the rule: $b x: t \mapsto b \wedge x(t)$ for $t \in \operatorname{dom}(x)$. Then $b x \in \mathbb{V}^{(B)}$. Moreover, for all $x, y \in \mathbb{V}^{(B)}$ we have $\llbracket x \in b y \rrbracket=b \wedge \llbracket x \in y \rrbracket$ and $\llbracket b x=b y \rrbracket=b \rightarrow \llbracket x=y \rrbracket$.

The checking of the first equality consists in the straightforward calculation of truth values and application on the infinite distributive law:

$$
\llbracket x \in b y \rrbracket=\bigvee_{t \in \operatorname{dom}(b y)}(b y)(t) \wedge \llbracket t=x \rrbracket=b \wedge \bigvee_{t \in \operatorname{dom}(y)} y(t) \wedge \llbracket t=x \rrbracket=b \wedge \llbracket x \in y \rrbracket .
$$

The second identity uses the rules of inference of 2.2 :

$$
\begin{gathered}
\llbracket b x=b y \rrbracket=\bigwedge_{t \in \operatorname{dom}(b y)}(b y)(t) \rightarrow \llbracket t \in b x \rrbracket \wedge \bigwedge_{t \in \operatorname{dom}(b x)}(b x)(t) \rightarrow \llbracket t \in b y \rrbracket \\
=\bigwedge_{t \in \operatorname{dom}(y)}(b \wedge y(t)) \rightarrow(b \wedge \llbracket t \in x \rrbracket) \wedge \bigwedge_{t \in \operatorname{dom}(x)}(b \wedge x(t)) \rightarrow(b \wedge \llbracket t \in y \rrbracket) \\
=\bigwedge_{t \in \operatorname{dom}(y)}((b \wedge y(t)) \rightarrow b) \wedge((b \wedge y(t)) \rightarrow \llbracket t \in x \rrbracket) \\
\wedge \bigwedge_{t \in \operatorname{dom}(x)}((b \wedge x(t)) \rightarrow b) \wedge((b \wedge x(t)) \rightarrow \llbracket t \in y \rrbracket) \\
=\bigwedge_{t \in \operatorname{dom}(y)} b \rightarrow(y(t) \rightarrow \llbracket t \in x \rrbracket) \wedge \bigwedge_{t \in \operatorname{dom}(x)} b \rightarrow(x(t) \rightarrow \llbracket t \in y \rrbracket)=b \rightarrow \llbracket x=y \rrbracket .
\end{gathered}
$$

(5) Take $x \in \mathbb{V}^{(B)}$ and define $\bar{x} \in \mathbb{V}^{(B)}$ by the rules $\operatorname{dom}(\bar{x}):=\operatorname{dom}(x)$ and $\bar{x}(t):=\llbracket t \in x \rrbracket$ for all $t \in \operatorname{dom}(x)$. Then $\mathbb{V}^{(B)} \models x=\bar{x}$ as shown by the easy calculations:

$$
\begin{gathered}
\llbracket x=\bar{x} \rrbracket=\bigwedge_{t \in \operatorname{dom}(x)} x(t) \rightarrow \llbracket t \in \bar{x} \rrbracket \wedge \bigwedge_{t \in \operatorname{dom}(\bar{x})} \llbracket t \in x \rrbracket \rightarrow \llbracket t \in x \rrbracket \\
=\bigwedge_{t \in \operatorname{dom}(x)} x(t) \rightarrow\left(\bigvee_{u \in \operatorname{dom}(\bar{x})} \bar{x}(u) \wedge \llbracket u=t \rrbracket\right) \geq \bigwedge_{t \in \operatorname{dom}(x)} x(t) \rightarrow \llbracket t \in x \rrbracket=\mathbb{1} .
\end{gathered}
$$

(6) Take a partition of unity $\left(b_{\xi}\right)_{\xi \in \Xi} \subset B$ and a family $\left(x_{\xi}\right)_{\xi \in \Xi} \subset \mathbb{V}^{(B)}$. Put

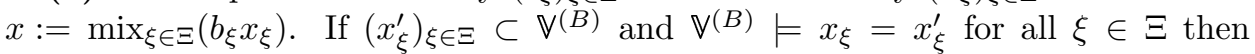
$\mathbb{V}^{(B)} \models x=\operatorname{mix}_{\xi \in \Xi}\left(b_{\xi} x_{\xi}^{\prime}\right)$. Indeed, let $x^{\prime}:=\operatorname{mix}_{\xi \in \Xi}\left(b_{\xi} x_{\xi}^{\prime}\right)$. It is then easy to see that $b_{\xi} \leq \llbracket x_{\xi}=x_{\xi}^{\prime} \rrbracket \wedge \llbracket x_{\xi}=x \rrbracket \wedge \llbracket x_{\xi}^{\prime}=x^{\prime} \rrbracket \leq \llbracket x=x^{\prime} \rrbracket$.

(7) If $y \in \mathbb{V}^{(B)}$ is such that $\operatorname{dom}(y)=\operatorname{dom}(x)$ and $y(t):=\bigvee_{\xi \in \Xi} b_{\xi} \wedge \llbracket t \in x_{\xi} \rrbracket$ for all $t \in \operatorname{dom}(y))$, then $\mathbb{V}^{(B)} \models x=y$.

(8) Given $b \in B$ and $x \in \mathbb{V}^{(B)}$, observe that $\llbracket b x=x \rrbracket=b \vee \llbracket x=\varnothing \rrbracket$ and $\llbracket b x=\varnothing \rrbracket=\neg b \vee \llbracket x=\varnothing \rrbracket$. In particular, $\mathbb{V}^{(B)} \models b x=\operatorname{mix}\{b x, \neg b \varnothing\}$.

(9) Let $\left(b_{\xi}\right)$ be a partition of unity in $B$ and let a family $\left(x_{\xi}\right) \subset \mathbb{V}^{(B)}$ be such that $\mathbb{V}^{(B)} \models x_{\xi} \neq x_{\eta}$ for all $\xi \neq \eta$. Then there is a member $x$ of $\mathbb{V}^{(B)}$ satisfying $\llbracket x=$ $x_{\xi} \rrbracket=b_{\xi}$ for all $\xi$. Indeed, put $x:=\operatorname{mix}\left(b_{\xi} x_{\xi}\right)$ and $a_{\xi}:=\llbracket x=x_{\xi} \rrbracket$. By hypothesis 
$a_{\xi} \wedge a_{\eta}=\llbracket x=x_{\xi} \rrbracket \wedge \llbracket x_{\eta}=x \rrbracket \leq \neg \llbracket x_{\xi} \neq x_{\eta} \rrbracket=\mathbb{0}$ for $\xi \neq \eta$. Moreover, $b_{\xi} \leq a_{\xi}$ for all $\xi$ by the properties of mixing. Hence, $\left(a_{\xi}\right)$ is a partition of unity in $B$ too. On the other hand, $\neg b_{\xi}=\bigvee_{\eta \neq \xi} b_{\eta} \leq \bigvee_{\eta \neq \xi} a_{\eta}=\neg a_{\xi}$ and $\neg b_{\xi} \leq \neg a_{\xi} \rightarrow b_{\xi} \geq a_{\xi}$. So, $\left(b_{\xi}\right)$ and $\left(a_{\xi}\right)$ present the same partition of unity.

(10) Consider some formulas $\varphi(x)$ and $\psi(x)$ of ZFC. Assume that $\llbracket \varphi\left(u_{0}\right) \rrbracket=\mathbb{1}$ for some $u_{0} \in \mathbb{V}^{(B)}$. Then

$$
\begin{aligned}
& \llbracket(\forall x)(\varphi(x) \rightarrow \psi(x)) \rrbracket=\bigwedge\left\{\llbracket \psi(u) \rrbracket \mid u \in \mathbb{V}^{(B)}, \llbracket \varphi(u) \rrbracket=\mathbb{1}\right\}, \\
& \llbracket(\exists x)(\varphi(x) \wedge \psi(x)) \rrbracket=\bigvee\left\{\llbracket \psi(u) \rrbracket \mid u \in \mathbb{V}^{(B)}, \llbracket \varphi(u) \rrbracket=\mathbb{1}\right\} .
\end{aligned}
$$

These formulas reveal the "mosaic" mechanisms of truth verification in $\mathbb{V}^{(B)}$.

3.3. Functional realization. Let $Q$ be the Stone space of a complete Boolean algebra $B$. Denote by $\mathfrak{U}$ the (separated) Boolean valued universe $\mathbb{V}^{(B)}$. Given $q \in Q$, define the equivalence $\sim_{q}$ on the class $\mathfrak{U}$ as follows: $u \sim_{q} v \leftrightarrow q \in \llbracket u=v \rrbracket$. Consider the bundle

$$
V^{Q}:=\left\{\left(q, \sim_{q}(u)\right) \mid q \in Q, u \in \mathfrak{U}\right\}
$$

and agree to denote the pair $\left(q, \sim_{q}(u)\right)$ by $\widehat{u}(q)$. Clearly, for every $u \in \mathfrak{U}$ the mapping $\widehat{u}: q \mapsto \widehat{u}(q)$ is a section of $V^{Q}$. Note that to each $x \in V^{Q}$ there are $u \in \mathfrak{U}$ and $q \in Q$ satisfying $\widehat{u}(q)=x$. Moreover, we have $\widehat{u}(q)=\widehat{v}(q)$ if and only if $q \in \llbracket u=v \rrbracket$.

Make each fiber $V^{q}$ of $V^{Q}$ into an algebraic system of signature $\{\in\}$ by letting $V^{q} \models x \in y \leftrightarrow q \in \llbracket u \in v \rrbracket$, where $u, v \in \mathfrak{U}$ are such that $\widehat{u}(q)=x$ and $\widehat{v}(q)=y$. Clearly, this definition is sound. Indeed, if $\widehat{u}_{1}(q)=x$ and $\widehat{v}_{1}(q)=y$ for another pair of elements $u_{1}$ and $v_{1}$ then the claims of the memberships $q \in \llbracket u \in v \rrbracket$ and $q \in \llbracket u_{1} \in v_{1} \rrbracket$ are equivalent.

It is easy to see that the class of the sets $\{\widehat{u}(A) \mid u \in \mathfrak{U}\}$, with $A$ a clopen subset of $Q$, is a base for some topology on $V^{Q}$. This enables us to view $V^{Q}$ as a continuous bundle called a continuous polyverse. By a continuous section of $V^{Q}$ we mean a section that is a continuous function. Denote by $\mathfrak{C}$ the class of all continuous sections of $V^{Q}$.

The mapping $u \mapsto \widehat{u}$ is a bijection between $\mathfrak{U}$ and $\mathfrak{C}$ in which we can clearly find grounds for a convenient functional realization of the Boolean valued universe $\mathbb{V}^{(B)}$. The details of this universal construction due to Gutman and Losenkov are presented in [8, Ch. 6].

3.4. Socialization. Mathematics of the twentieth century exhibited many examples of the achievements obtained by socialization of objects and problems; i.e., their inclusion into a class of similar objects or problems. ${ }^{3}$ Boolean valued models gain a natural status within category theory. The idea of a variable set becomes

\footnotetext{
${ }^{3}$ Hilbert said in his report 3: "In dealing with mathematical problems, specialization plays, as I believe, a still more important part than generalization. Perhaps in most cases where we seek in vain the answer to a question, the cause of the failure lies in the fact that problems simpler and easier than the one in hand have been either not at all or incompletely solved. All depends, then, on finding out these easier problems, and on solving them by means of devices as perfect as possible and of concepts capable of generalization. This rule is one of the most important levers for overcoming mathematical difficulties and it seems to me that it is used almost always, though perhaps unconsciously."
} 
a cornerstone of the categorical analysis of logic which is accomplished in topos theory [4.

3.5. Distant modeling. The properties of a Boolean valued universe reflects a new conception of modeling which may be referred to as extramural or distant modeling. We will explain the essence of this conception by comparing it with the traditional approaches.

Encountering two classical models of the same theory, we usually seek for a bijection between the universes of the models. If we manage to establish such a bijection so as the predicates and operations translate faithfully from one model to the other then we speak about isomorphism between the models. Consequently, this conception of isomorphism implies an explicit comparison of the models which consists in witnessing some bijection between their universes of discourse.

Imagine that we are physically unable to compare the models pointwise. Happily, we take an opportunity to exchange information with the owner of the other model by using some means of communication, e.g., by having long-distance calls. While communicating, we easily learn that our partner uses his model to operate on some objects that are the namesakes of ours, i.e., sets, membership, etc. Since we are interested in ZFC, we ask him whether or not the axioms of ZFC are satisfied in his model. Manipulating the model, he returns a positive answer. After checking that he uses the same inference rules as we do, we cannot help but acknowledge his model to be a model of the theory we are all investigating. It is worth noting that this conclusion still leaves unknown for us the objects that make up his universe and the procedures he uses to distinguish between true and false propositions about these objects.

The novelty of distant modeling resides in our refusal to identify the universes of objects and admittance of some a priori unknown procedures of verification of propositions.

3.6. Technology. To prove the relative consistency of some set-theoretic propositions we use a Boolean valued universe $\mathbb{V}^{(B)}$ as follows: Let $\mathscr{T}$ and $\mathscr{S}$ be some enrichments of Zermelo-Fraenkel theory ZF (without choice). Assume that the consistency of ZF implies the consistency of $\mathscr{S}$. Assume further that we can define $B$ so that $\mathscr{S} \models$ " $B$ is a complete Boolean algebra" and $\mathscr{S} \models \llbracket \varphi \rrbracket=\mathbb{1}$ for every axiom $\varphi$ of $\mathscr{T}$. Then the consistency of ZF implies the consistency of $\mathscr{T}$. That is how we use $\mathbb{V}^{(B)}$ in foundational studies.

Other possibilities for applying $\mathbb{V}^{(B)}$ base on the fact that irrespective of the choice of a Boolean algebra $B$, the universe is an arena for testing an arbitrary mathematical event. By the principles of transfer and maximum, every $\mathbb{V}^{(B)}$ has the objects that play the roles of numbers, groups, Banach spaces, manifolds, and whatever constructs of mathematics that are already introduced into practice or still remain undiscovered. These objects may be viewed as some nonstandard realizations of the relevant originals.

All celebrated and not so popular theorems acquire interpretations for the members of $\mathbb{V}^{(B)}$, attaining the top truth value. We thus obtain a new technology of comparison between the interpretations of mathematical facts in the universes over various complete Boolean algebras. Developing the relevant tools is the crux of Boolean valued analysis. 


\section{Ascending and Descending}

No comparison is feasible without some dialog between $\mathbb{V}$ and $\mathbb{V}^{(B)}$. We need some sufficiently convenient mathematical toolkit for the comparative analysis of the interpretations of the concepts and facts of mathematics in various models. The relevant technique of ascending and descending bases on the operations of the canonical embedding, descent, and ascent to be addressed right away. We start with the canonical embedding of the von Neumann universe.

4.1. The canonical embedding. Given $x \in \mathbb{V}$, denote by $x^{\wedge}$ the standard name of $x$ in $\mathbb{V}^{(B)}$; i.e., the member of $\mathbb{V}^{(B)}$ that is defined by recursion as follows:

$$
\varnothing^{\wedge}:=\varnothing, \quad \operatorname{dom}\left(x^{\wedge}\right):=\left\{y^{\wedge} \mid y \in x\right\}, \quad \operatorname{im}\left(x^{\wedge}\right):=\{\mathbb{1}\} .
$$

Note a few properties of the mapping $x \mapsto x^{\wedge}$ which we will use in the sequel.

(1) If $x \in \mathbb{V}$ and $\varphi$ is a formula of ZFC then

$$
\begin{aligned}
& \llbracket\left(\exists y \in x^{\wedge}\right) \varphi(y) \rrbracket=\bigvee\left\{\llbracket \varphi\left(z^{\wedge}\right) \rrbracket \mid z \in x\right\}, \\
& \llbracket\left(\forall y \in x^{\wedge}\right) \varphi(y) \rrbracket=\bigwedge\left\{\llbracket \varphi\left(z^{\wedge}\right) \rrbracket \mid z \in x\right\} .
\end{aligned}
$$

(2) If $x$ and $y$ are members of $\mathbb{V}$ then we see by transfinite induction that

$$
x \in y \leftrightarrow \mathbb{V}^{(B)} \models x^{\wedge} \in y^{\wedge}, \quad x=y \leftrightarrow \mathbb{V}^{(B)} \models x^{\wedge}=y^{\wedge} .
$$

In other words, the standard name may be viewed as the embedding functor from $\mathbb{V}$ to $\mathbb{V}^{(B)}$. Undoubtedly, the standard name sends $\mathbb{V}$ onto $\mathbb{V}^{(2)}$, which is distinguished by the following proposition:

(3) $\left(\forall u \in \mathbb{V}^{(2)}\right)(\exists ! x \in \mathbb{V}) \mathbb{V}^{(B)} \models u=x^{\wedge}$.

(4) A formula is restricted provided that each bound variable enters this formula under the sign of some restricted quantifier; i.e., a quantifier ranging over a set. Strictly speaking, each bound variable must be restricted by a quantifier of the form $(\forall x \in y)$ or $(\exists x \in y)$ for some $y$.

Restricted Transfer Principle. Given a restricted formula $\varphi$ of ZFC and a collection $x_{1}, \ldots, x_{n} \in \mathbb{V}$, the following holds:

$$
\varphi\left(x_{1}, \ldots, x_{n}\right) \leftrightarrow \mathbb{V}^{(B)} \models \varphi\left(x_{1}^{\wedge}, \ldots, x_{n}^{\wedge}\right) .
$$

Working further in the separated universe $\overline{\mathbb{V}}^{(B)}$, we agree to preserve the symbol $x^{\wedge}$ for the distinguished member of the class corresponding to $x$.

(5) By way of example, we mention the useful consequence of the restricted transfer principle:

$$
\begin{gathered}
\text { " } \Phi \text { is a correspondence from } x \text { to } y " \\
\leftrightarrow \mathbb{V}^{(B)} \models " \Phi^{\wedge} \text { is a correspondence from } x^{\wedge} \text { to } y^{\wedge} " ;
\end{gathered}
$$

" $f$ is a function from $x$ to $y " \leftrightarrow \mathbb{V}^{(B)} \models " ~ f \wedge$ is a function from $x^{\wedge}$ to $y^{\wedge} "$

(in this event, $f(a)^{\wedge}=f^{\wedge}\left(a^{\wedge}\right)$ for all $\left.a \in x\right)$. Therefore, the standard name may be considered as a covariant functor from the category of sets and mappings (or correspondences) inside $\mathbb{V}$ to an appropriate subcategory $\mathbb{V}^{(2)}$ inside the separated universe $\mathbb{V}^{(B)}$.

(6) A set $x$ is finite provided that $x$ coincides with the range of a function on a finite ordinal. We express this symbolically as $\operatorname{Fin}(x)$. Hence, Fin $(x):=$ $(\exists n)(\exists f)(n \in \omega \wedge \operatorname{Fnc}(f) \wedge \operatorname{dom}(f)=n \wedge \operatorname{im}(f)=x)$ where $\omega:=\{0,1,2, \ldots\}$ as usual. The above formula clearly fails to be restricted. However, the transformation 
of finite sets proceeds happily under the canonical embedding. Indeed, let $\mathscr{P}_{\text {Fin }}(x)$ stand for the class of finite subsets of $x$; i.e., $\mathscr{P}_{\text {Fin }}(x):=\{y \in \mathscr{P}(x) \mid \operatorname{Fin}(y)\}$. Then $\mathbb{V}^{(B)} \models \mathscr{P}_{\text {Fin }}(x)^{\wedge}=\mathscr{P}_{\text {Fin }}\left(x^{\wedge}\right)$ for all $X$. Since the formula specifying the powerset of a set $x$ is unrestricted, in general we may claim only that $\llbracket \mathscr{P}(x)^{\wedge} \subset \mathscr{P}\left(x^{\wedge}\right) \rrbracket=\mathbb{1}$.

(7) Let $\rho$ be an automorphism of a complete Boolean algebra $B$, and let $\psi_{\rho}$ be the member of $\vee^{(B)}$ defined by the function $\left\{\left(b^{\wedge}, \rho(b)\right) \mid b \in B\right\}$. Then

(a) $\rho(b)=\llbracket b^{\wedge} \in \psi_{\rho} \rrbracket$ for all $b \in B$;

(b) $\llbracket A^{\wedge} \subset \psi_{\rho} \rightarrow(\bigwedge A)^{\wedge} \in \psi_{\rho} \rrbracket=\mathbb{1}$ for $A \subset B$ if and only if $\rho(\bigwedge A)=\bigwedge \rho(A)$;

(c) $\llbracket \psi_{\rho}$ is an ultrafilter on $B^{\wedge} \rrbracket=\mathbb{1}$.

(8) Let $\pi$ be a homomorphism of $B$ to a complete Boolean algebra $C$. By recursion on the relation $y \in \operatorname{dom}(x)$ we can define the mapping $\pi^{*}: \mathbb{V}^{(B)} \rightarrow \mathbb{V}^{(C)}$ such that $\operatorname{dom}\left(\pi^{*} x\right):=\left\{\pi^{*} y \mid y \in \operatorname{dom}(x)\right\}$ and $\pi^{*} x: v \mapsto \bigvee\{\pi(x(z)) \mid z \in$ $\left.\operatorname{dom}(x), \pi^{*} z=v\right\}$. If $\pi$ is injective then so is $\pi^{*}$. Moreover, $\pi^{*} x: \pi^{*} y \mapsto \pi(x(y))$ for all $y \in \operatorname{dom}(x)$.

Let $\mathfrak{U}$ be an ultrafilter on a Boolean algebra $B$, and let $\mathfrak{U}^{\prime}$ be the dual ideal; namely, $\mathfrak{U}^{\prime}:=\{\neg b \mid b \in \mathfrak{U}\}$. Then the factor-algebra $B / \mathfrak{U}^{\prime}$ has two members and we may identify it with the two-point Boolean algebra $2:=\{\mathbb{0}, \mathbb{1}\}$. The factorhomomorphism $\pi: B \rightarrow 2$ is not complete in general; i.e., $\pi$ fails to preserve all joins and meets. This prevents us from interconnecting the truth values on $\mathbb{V}^{(B)}$ and $\mathbb{V}^{(2)}$. However, if $\pi$ is complete (i.e., $\mathfrak{U}$ is a principal ultrafilter), then for every formula $\varphi\left(x_{1}, \ldots, x_{n}\right)$ and every tuple $u_{1}, \ldots, u_{n} \in \mathbb{V}^{(B)}$ we have

$$
\mathbb{V}^{(\mathcal{2})} \models \varphi\left(\pi^{*} u_{1}, \ldots, \pi^{*} u_{n}\right) \leftrightarrow \llbracket \varphi\left(u_{1}, \ldots, u_{n}\right) \rrbracket \in \mathfrak{U},
$$

since the formulas $\pi(b)=\mathbb{1}$ and $b \in \mathfrak{U}$ are equivalent for $b \in B$..

(9) Given an ultrafilter $\mathfrak{U}$, we can use factorization to construct a model that differs from $\mathbb{V}^{(\mathfrak{2})}$. To this end, we introduce in $\mathbb{V}^{(B)}$ the relation $\sim_{\mathfrak{U}}$ by the rule $\sim_{\mathfrak{U}}:=\left\{(x, y) \in \mathbb{V}^{(B)} \times \mathbb{V}^{(B)} \mid \llbracket x=y \rrbracket \in \mathfrak{U}\right\}$. Clearly, $\sim_{\mathfrak{U}}$ is an equivalence on $\mathbb{V}^{(B)}$. Denote by $\mathbb{V}^{(B)} / \mathfrak{U}$ the factor-class of $\mathbb{V}^{(B)}$ by $\sim_{\mathfrak{U}}$ and furnish it with the relation $\epsilon_{\mathfrak{U}}:=\left\{(\widetilde{x}, \widetilde{y}) \mid x, y \in \mathbb{V}^{(B)}, \llbracket x \in y \rrbracket \in \mathfrak{U}\right\}$, where $x \mapsto \widetilde{x}$ is the canonical factormapping from $\mathbb{V}^{(B)}$ to $\mathbb{V}^{(B)} / \mathfrak{U}$. We can show that $\mathbb{V}^{(B)} / \mathfrak{U}=\varphi\left(\widetilde{x}_{1}, \ldots, \widetilde{x}_{n}\right) \leftrightarrow$ $\llbracket \varphi\left(x_{1}, \ldots, x_{n}\right) \rrbracket \in \mathfrak{U}$ for $x_{1}, \ldots, x_{n} \in \mathbb{V}^{(B)}$ and a formula $\varphi$ of ZFC.

4.2. Descent. Given an arbitrary member $x$ of a (separated) Boolean valued universe $\mathbb{V}^{(B)}$, define the descent $x \downarrow$ of $x$ as follows:

$$
x \downarrow:=\left\{y \in \mathbb{V}^{(B)} \mid \llbracket y \in x \rrbracket=\mathbb{1}\right\} .
$$

We list the simplest properties of descending:

(1) The class $x \downarrow$ is a set; i.e., $x \downarrow \in \mathbb{V}$ for all $x \in \mathbb{V}^{(B)}$. If $\llbracket x \neq \varnothing \rrbracket=\mathbb{1}$ then $x \downarrow$ is a nonempty set.

(2) Let $z \in \mathbb{V}^{(B)}$ and $\llbracket z \neq \varnothing \rrbracket=\mathbb{1}$. Then for every formula $\varphi$ of ZFC we have

$$
\begin{aligned}
& \llbracket(\forall x \in z) \varphi(x) \rrbracket=\bigwedge\{\llbracket \varphi(x) \rrbracket \mid x \in z \downarrow\}, \\
& \llbracket(\exists x \in z) \varphi(x) \rrbracket=\bigvee\{\llbracket \varphi(x) \rrbracket \mid x \in z \downarrow\} .
\end{aligned}
$$

Moreover, there is some $x_{0} \in z \downarrow$ satisfying $\llbracket \varphi\left(x_{0}\right) \rrbracket=\llbracket(\exists x \in z) \varphi(x) \rrbracket$.

(3) Let $\Phi$ be a correspondence from $X$ to $Y$ inside $\mathbb{V}^{(B)}$. In other words, $\Phi$, $X$, and $Y$ are members of $\mathbb{V}^{(B)}$, while $\llbracket \Phi \subset X \times Y \rrbracket=\mathbb{1}$. Then there is a unique correspondence $\Phi \downarrow$ from $X \downarrow$ to $Y \downarrow$ such that $\Phi \downarrow(A \downarrow)=\Phi(A) \downarrow$ for every nonempty 
subset $A$ of $X$ inside $\mathbb{V}^{(B)}$. The resulting correspondence $\Phi \downarrow$ from $X \downarrow$ to $Y \downarrow$ is the descent of $\Phi$ from $X$ to $Y$ inside $\mathbb{V}^{(B)}$.

(4) The descent of the composite of correspondences inside $\mathbb{V}^{(B)}$ is the composite of their descents: $(\Psi \circ \Phi) \downarrow=\Psi \downarrow \circ \Phi \downarrow$.

(5) If $\Phi$ is a correspondence inside $\mathbb{V}^{(B)}$ then $\left(\Phi^{-1}\right) \downarrow=(\Phi \downarrow)^{-1}$.

(6) Let $\operatorname{Id}_{X}$ be the identity mapping inside $\mathbb{V}^{(B)}$ of some $X \in \mathbb{V}^{(B)}$. Then $\left(\operatorname{Id}_{X}\right) \downarrow=\operatorname{Id}_{X \downarrow}$.

(7) Assume that $\llbracket f: X \rightarrow Y \rrbracket=\mathbb{1}$, with $X, Y, f \in \mathbb{V}^{(B)}$; i.e., $f$ is a funstion from $X$ to $Y$ inside $\mathbb{V}^{(B)}$. Then $f \downarrow$ is the unique mapping from $X \downarrow$ to $Y \downarrow$ such that $\llbracket f \downarrow(x)=f(x) \rrbracket=\mathbb{1}$ for all $x \in X \downarrow$.

By (1)-(7), we may view the descent as a functor from the category of $B$-valued sets and mappings (correspondences) to the usual category of sets and mappings (correspondences) in the sense of $\mathbb{V}$.

(8) Given $x_{1}, \ldots, x_{n} \in \mathbb{V}^{(B)}$, denote by $\left(x_{1}, \ldots, x_{n}\right)^{B}$ the corresponding $n$-tuple inside $\mathbb{V}^{(B)}$. Assume that $P$ is a $n$-ary relation on $X$ inside $\mathbb{V}^{(B)}$; i.e., $X, P \in \mathbb{V}^{(B)}$ and $\llbracket P \subset X^{n^{\wedge}} \rrbracket=\mathbb{1}$, with $n \in \omega$. Then there is a $n$-ary relation $P^{\prime}$ on $X \downarrow$ such that

$$
\left(x_{1}, \ldots, x_{n}\right) \in P^{\prime} \leftrightarrow \llbracket\left(x_{1}, \ldots, x_{n}\right)^{B} \in P \rrbracket=\mathbb{1} .
$$

Slightly abusing notation, we will denote $P^{\prime}$ by the already-occupied symbol $P \downarrow$ and call $P \downarrow$ the descent of $P$.

4.3. Ascent. Assume that $x \in \mathbb{V}$ and $x \subset \mathbb{V}^{(B)}$; i.e., let $x$ be some set composed of $B$-valued sets or, in other words, $x \in \mathscr{P}\left(\mathbb{V}^{(B)}\right)$. Put $\varnothing \uparrow:=\varnothing$, while $\operatorname{dom}(x \uparrow):=x$ and $\operatorname{im}(x \uparrow):=\{\mathbb{1}\}$ in case $x \neq \varnothing$. The member $x \uparrow$ of the (separated) universe $\mathbb{V}^{(B)}$; i.e., the distinguished representative of the class $\left\{y \in \mathbb{V}^{(B)} \mid \llbracket y=x \uparrow \rrbracket=\mathbb{1}\right\}$, is the ascent of $x$.

(1) For all $x \in \mathscr{P}\left(\mathbb{V}^{(B)}\right)$ and every formula $\varphi$, the following are valid:

$$
\llbracket(\forall z \in x \uparrow) \varphi(z) \rrbracket=\bigwedge_{y \in x} \llbracket \varphi(y) \rrbracket, \quad \llbracket(\exists z \in x \uparrow) \varphi(z) \rrbracket=\bigvee_{y \in x} \llbracket \varphi(y) \rrbracket
$$

Ascending the correspondence $\Phi \subset X \times Y$, we should keep in mind that the domain of departure of $\Phi$, which is $X$, may differ from the domain of $\Phi$, which is $\operatorname{dom}(\Phi):=\{x \in X \mid \Phi(x) \neq \varnothing\}$. This circumstance is immaterial for our nearest goals. Therefore, speaking about ascents, we will always imply the total correspondences $\Phi$ satisfying $\operatorname{dom}(\Phi)=X$.

(2) Assume that $X, Y, \Phi \in \mathbb{V}^{(B)}$, and $\Phi$ is a correspondence from $X$ to $Y$. There is a unique correspondence $\Phi \uparrow$ from $X \uparrow$ to $Y \uparrow$ inside $\mathbb{V}^{(B)}$ such that the equality $\Phi \uparrow(A \uparrow)=\Phi(A) \uparrow$ holds for every subset $A$ of $\operatorname{dom}(\Phi)$ if and only if $\Phi$ is extensional; i.e., $\Phi$ enjoys the property

$$
y_{1} \in \Phi\left(x_{1}\right) \rightarrow \llbracket x_{1}=x_{2} \rrbracket \leq \bigvee_{y_{2} \in \Phi\left(x_{2}\right)} \llbracket y_{1}=y_{2} \rrbracket
$$

for $x_{1}, x_{2} \in \operatorname{dom}(\Phi)$. Moreover, $\Phi \uparrow=\Phi^{\prime} \uparrow$, with $\Phi^{\prime}:=\left\{(x, y)^{B} \mid(x, y) \in \Phi\right\}$. The member $\Phi \uparrow$ of $\mathbb{V}^{(B)}$ is the ascent of $\Phi$.

(3) The composite of extensional correspondences is extensional too. Moreover, the ascent of the composite is the composite of the ascents inside $\mathbb{V}^{(B)}$; i.e., if $\operatorname{dom}(\Psi) \supset \operatorname{im}(\Phi)$ then $\mathbb{V}^{(B)} \models(\Psi \circ \Phi) \uparrow=\Psi \uparrow \circ \Phi \uparrow$.

Note also that if $\Phi$ and $\Phi^{-1}$ are extensional then $(\Phi \uparrow)^{-1}=\left(\Phi^{-1}\right) \uparrow$. However, the extensionality of $\Phi$ does not guarantee the extensionality of $\Phi^{-1}$ in general. 
(4) It is worth mentioning that if some extensional correspondence $f$ is a function from $X$ to $Y$ then the ascent $f \uparrow$ of $f$ will be a function from $X \uparrow$ to $Y \uparrow$. In this event, we may write down the extensionality property as follows: $\llbracket x_{1}=x_{2} \rrbracket \leq$ $\llbracket f\left(x_{1}\right)=f\left(x_{2}\right) \rrbracket$ for all $x_{1}, x_{2} \in X$.

Given $X \subset \mathbb{V}^{(B)}$, we let the symbol $\operatorname{mix}(X)$ stand for the collection of all mixings like $\operatorname{mix}\left(b_{\xi} x_{\xi}\right)$, where $\left(x_{\xi}\right) \subset X$ and $\left(b_{\xi}\right)$ is an arbitrary partition of unity. The propositions to follow are called the arrow cancellation rules or the rules for ascending and descending. There are many nice reasons to refer to them simply as the Escher rules [6].

(5) Let $X$ and $X^{\prime}$ be two subsets of $\mathbb{V}^{(B)}$, and let $f: X \rightarrow X^{\prime}$ be extensional. Assume that $Y, Y^{\prime}, g \in \mathbb{V}^{(B)}$ enjoy the property $\llbracket Y \neq \varnothing \rrbracket=\llbracket g: Y \rightarrow Y^{\prime} \rrbracket=\mathbb{1}$. Then

$$
\begin{gathered}
X \uparrow \downarrow=\operatorname{mix}(X), \quad Y \downarrow \uparrow=Y ; \\
f \uparrow \downarrow=f, \quad g \downarrow \uparrow=g .
\end{gathered}
$$

(6) By analogy with 4.1(6), we easily infer the following convenient equality:

$$
\mathscr{P}_{\text {Fin }}(X \uparrow)=\left\{\theta \uparrow \mid \theta \in \mathscr{P}_{\text {Fin }}(X)\right\} \uparrow .
$$

We may generally assert inside $\mathbb{V}^{(B)}$ only that $\mathscr{P}(X \uparrow) \supset\{\theta \uparrow \mid \theta \in \mathscr{P}(X)\} \uparrow$.

4.4. Modified descents and ascents. Let $X \in \mathbb{V}$ and $X \neq \varnothing$; i.e., $X$ is a nonempty set. Denote by $\imath$ the canonical embedding $x \mapsto x^{\wedge}$ for $x \in X$. Then $\imath(X) \uparrow=X^{\wedge}$ and $X=\imath^{-1}\left(X^{\wedge} \downarrow\right)$. Using these equalities, we can translate the operations of ascending and descending to the case in which $\Phi$ is a correspondence from $X$ to $Y \downarrow$ and $\llbracket \Psi$ is a correspondence from $X^{\wedge}$ to $Y \rrbracket=\mathbb{1}$, where $Y \in \mathbb{V}^{(B)}$. Namely, we put $\Phi \uparrow:=(\Phi \circ \imath) \uparrow$ and $\Psi \downarrow:=\Psi \downarrow \circ \imath$. In this event, the member $\Phi \uparrow$ is the modified ascent of $\Phi$, while $\Psi \downarrow$ is the modified descent of $\Psi$. If the context prevents us from ambiguity then we will continue to speak simply about ascents and descents, using the usual arrows. Clearly, $\Psi \uparrow$ is the unique correspondence inside $\mathbb{V}^{(B)}$ enjoying the equality $\llbracket \Phi \uparrow\left(x^{\wedge}\right)=\Phi(x) \uparrow \rrbracket=\mathbb{1}$ for all $x \in X$. By analogy, $\Psi \downarrow$ is the unique correspondence from $X$ to $Y \downarrow$ such that the equality $\Psi \downarrow(x)=$ $\Psi\left(x^{\wedge}\right) \downarrow$ holds for all $x \in X$. If $\Phi:=f$ and $\Psi:=g$ are functions then the above equalities take the shape $\llbracket f \uparrow\left(x^{\wedge}\right)=f(x) \rrbracket=\mathbb{1}$ and $g \downarrow(x)=g\left(x^{\wedge}\right)$ for all $x \in X$.

\section{Elements of Boolean Valued Analysis}

Every Boolean valued universe has a complete mathematical toolkit containing the sets with arbitrary additional structures: groups, rings, algebras, etc. Descending the algebraic systems from Boolean valued models yields new objects with new properties, revealing many facts about their constructions and interrelations. Search into these interrelations is the content of Boolean valued analysis. In this survey we naturally confine exposition only to the basics of the relevant technique.

5.1. Boolean sets. We start with recognizing the simplest objects that descend from a Boolean valued universe.

(1) A Boolean set, or a set with $B$-structure, or simply a $B$-set is by definition a pair $(X, d)$, where $X \in \mathbb{V}, X \neq \varnothing$, and $d$ is a mapping from $X \times X$ to a Boolean algebra $B$ satisfying for all $x, y, z \in X$ the following conditions:

(a) $d(x, y)=\mathbb{0} \leftrightarrow x=y$;

(b) $d(x, y)=d(y, x)$

(c) $d(x, y) \leq d(x, z) \vee d(z, y)$. 
Each nonempty subset $\varnothing \neq X \subset \mathbb{V}^{(B)}$ provides an example of a $B$-set on assuming that $d(x, y):=\llbracket x \neq y \rrbracket=\neg \llbracket x=y \rrbracket$ for all $x, y \in X$. Another example arises if we furnish a nonempty set $X$ with the "discrete $B$-metric" $d$; i.e., on letting $d(x, y)=\mathbb{1}$ in case $x \neq y$ and $d(x, y)=\mathbb{0}$ in case $x=y$.

(2) Given an arbitrary Boolean algebra $D$, we may take as a $D$-metric the symmetric difference: $x \triangle y:=(x \wedge \neg y) \vee(y \wedge \neg x)$. We proceed now with a relevant construction. Let $\psi$ be an ultrafilter on a Bollean algebra $D$. Consider a Boolean set $\left(X, d_{X}\right)$ with some $D$-metric $d_{X}$. Introduce the binary relation $\sim_{\psi}$ on $X$ by the rule $(x, y) \in \sim_{\psi} \leftrightarrow \neg d_{X}(x, y) \in \psi$. From the definition of Boolean metric it follows that $\sim_{\psi}$ is an equivalence. Let $X / \sim_{\psi}$ stand for the factor-set of $X$ by $\sim_{\psi}$, and let $\pi_{X}: X \rightarrow X / \sim_{\psi}$ be the canonical factor-mapping. If we start with the Boolean set $(D, \Delta)$ then we see that $D / \sim_{\psi}$ is the two-point Boolean algebra 2. In this event there is a unique mapping $\bar{d}: X / \sim_{\psi} \rightarrow 2$ such that $\bar{d}\left(\pi_{X}(x), \pi_{X}(y)\right)=\pi_{D}(d(x, y))$ for all $x, y \in X$. Moreover, $\bar{d}$ is the discrete Boolean metric on $X / \sim_{\psi}$. If $d_{X}$ is the discrete metric on $X$ then $\sim_{\psi}=\operatorname{Id}$ and $X / \sim_{\psi}=X$.

(3) Let $(X, d)$ be some $B$-set. Assume that $\psi:=\psi_{\operatorname{Id}_{B}}$ is an ultrafilter constructed on $B^{\wedge}$ as in $4.1(7)$. By the restricted transfer principle $\left(X^{\wedge}, d^{\wedge}\right)$ is a $B^{\wedge}$-set inside $\mathbb{V}^{(B)}$. Put $\mathscr{X}:=X^{\wedge} / \sim_{\psi}$. In this event $\llbracket x^{\wedge} \sim_{\psi} y^{\wedge} \rrbracket=\neg d(x, y)$ for all $x, y \in X$. Consequently, there are a member $\mathscr{X}$ of $\mathbb{V}^{(B)}$ and an injection $\imath: X \rightarrow X^{\prime}:=\mathscr{X} \downarrow$ such that $d(x, y)=\llbracket \imath(x) \neq \imath(y) \rrbracket$ for all $x, y \in X$ and every $x^{\prime} \in X^{\prime}$ admits the representation $x^{\prime}=\operatorname{mix}_{\xi \in \Xi}\left(b_{\xi} \imath\left(x_{\xi}\right)\right)$, with $\left(x_{\xi}\right)_{\xi \in \Xi} \subset X$ and $\left(b_{\xi}\right)_{\xi \in \Xi}$ a partition of unity in $B$. The element $\mathscr{X}$ of $\mathbb{V}^{(B)}$ is the Boolean valued realization of the $B$-set $X$. If $X$ is a discrete $B$-set then $\mathscr{X}=X^{\wedge}$ and $\imath(x)=x^{\wedge}$ for all $x \in X$. If $X \subset \mathbb{V}^{(B)}$ then $\imath \uparrow$ is an injection from $X \uparrow$ to $\mathscr{X}$ inside $\mathbb{V}^{(B)}$.

(4) A mapping $f$ from a $B$-set $(X, d)$ to a $B$-set $\left(X^{\prime}, d^{\prime}\right)$ is contractive provided that $d^{\prime}(f(x), f(y)) \leq d(x, y)$ for all $x, y \in X$.

Assume that $X$ and $Y$ are some $B$-sets. Assume further that $\mathscr{X}$ and $\mathscr{Y}$ are the Boolean valued realizations of $X$ and $Y$, while $\imath: X \rightarrow \mathscr{X} \downarrow$ and $\jmath: Y \rightarrow \mathscr{Y} \downarrow$ are the corresponding injections. If $f: X \rightarrow Y$ is a contractive mapping then there is a unique member $g$ of $\mathbb{V}^{(B)}$ such that $\llbracket g: \mathscr{X} \rightarrow \mathscr{Y} \rrbracket=\mathbb{1}$ and $f=J^{-1} \circ g \downarrow \circ \imath$. We agree to let $\mathscr{X}:=\mathscr{F}^{\sim}(X):=X^{\sim}$ and $g:=\mathscr{F} \sim(f):=f^{\sim}$.

(5) The following hold:

(a) $\vee^{(B)} \models f(A)^{\sim}=f^{\sim}\left(A^{\sim}\right)$ for $A \subset X$;

(b) If $g: Y \rightarrow Z$ is a contractive mapping then so is $g \circ f$ and, moreover, $\mathbb{V}^{(B)} \models(g \circ f)^{\sim}=g^{\sim} \circ f^{\sim}$

(c) $\mathbb{V}(B) \models " f \sim$ is an injection" if and only if $f$ is a $B$-isometry;

(d) $\vee^{(B)} \models " f \sim$ is a surjection" if and only if

$$
(\forall y \in Y) \bigvee\{d(f(x), y) \mid x \in X\}=\mathbb{1} .
$$

(6) Consider a $B$-set $(X, d)$. Given $A \subset X$, denote by $\operatorname{mix}(A)$ the set of all mixing of the members of $A$. If $\operatorname{mix}(A)=A$ then we say that $A$ is a cyclic subset of $X$. The intersection of all cyclic sets including $A$ is denoted by $\operatorname{cyc}(A)$. A Boolean set $X$ is universally complete or extended provided that $X$ contains all mixings $\operatorname{mix}\left(b_{\xi} x_{\xi}\right)$ of arbitrary families $\left(x_{\xi}\right) \subset X$ by all partitions of unity $\left(b_{\xi}\right) \subset B$. In case these mixings exist for finite families, $X$ is finitely complete or decomposable. It can be shown that if $X$ is a universally complete $B$-set then $\operatorname{mix}(A)=\operatorname{cyc}(A)$ for every $A \subset X$. A cyclic subset of a $B$-set may fail to be universally complete. At the 
same time, each cyclic subset of $\bigvee^{(B)}$, furnished with the canonical $B$-metric, is a universally complete $B$-set.

5.2. Algebraic $\boldsymbol{B}$-systems. Recall that by a signature we mean the 3 -tuple $\sigma:=(F, P, \mathfrak{a})$, where $F$ and $P$ are some (possibly empty) sets, while $\mathfrak{a}$ is a mapping from $F \cup P$ to $\omega$. If $F$ and $P$ are finite then $\sigma$ is a finite signature. In applications we usually deal with the algebraic systems of finite signature.

By a $n$-ary operation and $n$-ary predicate on a $B$-set $A$ we mean some contractive mappings $f: A^{n} \rightarrow A$ and $p: A^{n} \rightarrow B$. Recall that $f$ and $p$ are contractive provided that

$$
\begin{aligned}
& d\left(f\left(a_{0}, \ldots, a_{n-1}\right), f\left(a_{0}^{\prime}, \ldots, a_{n-1}^{\prime}\right)\right) \leq \bigvee_{k=0}^{n-1} d\left(a_{k}, a_{k}^{\prime}\right), \\
& \triangle\left(p\left(a_{0}, \ldots, a_{n-1}\right), p\left(a_{0}^{\prime}, \ldots, a_{n-1}^{\prime}\right)\right) \leq \bigvee_{k=0}^{n-1} d\left(a_{k}, a_{k}^{\prime}\right)
\end{aligned}
$$

for all $a_{0}, a_{0}^{\prime}, \ldots, a_{n-1}, a_{n-1}^{\prime} \in A$, where $d$ is the $B$-metric on $A$.

The above definitions depend explicitly on $B$ and so it would be wise to speak of $B$-operations, $B$-predicates, etc. However, it is wiser to simplify and economize whenever this involves no confusion.

An algebraic $B$-system $\mathfrak{A}$ of signature $\sigma$ is by definition a pair $(A, \nu)$, where $A$ is a nonempty $B$-set, the carrier, or underlying set, or universe of $\mathfrak{A}$, while $\nu$ is a mapping such that

(a) $\operatorname{dom}(\nu)=F \cup P$;

(b) $\nu(f)$ is an $\mathfrak{a}(f)$-ary operation on $A$ for all $f \in F$;

(c) $\nu(p)$ is an $\mathfrak{a}(p)$-ary predicate on $A$ for all $p \in P$.

We call $\nu$ the interpretation of $\mathfrak{A}$ and write $f^{\nu}$ and $p^{\nu}$ instead of $\nu(f)$ and $\nu(p)$. The signature of an algebraic $B$-system $\mathfrak{A}:=(A, \nu)$ is often denoted by $\sigma(\mathfrak{A})$ and the carrier $A$ of $\mathfrak{A}$, by $|\mathfrak{A}|$.

An algebraic $B$-system $\mathfrak{A}:=(A, \nu)$ is universally complete or finitely complete provided that so is the carrier of $A$. Since $A^{0}=\{\varnothing\}$, the 0 -ary operations and predicates on $A$ are mappings from $\{\varnothing\}$ to $A$ and $B$ respectively. We will identify the mapping $g:\{\varnothing\} \rightarrow A \cup B$ with the set $g(\varnothing)$. Each 0 -ary operation on $A$ transforms in this fashion into a unigue member of $A$. Similarly, the set of all 0-ary predicates on $A$ becomes a subset of the Boolean algebra $B$.

If $F:=\left\{f_{1}, \ldots, f_{n}\right\}$ and $P:=\left\{p_{1}, \ldots, p_{m}\right\}$ then an algebraic $B$-system of signature $\sigma$ is written as $\left(A, \nu\left(f_{1}\right), \ldots, \nu\left(f_{n}\right), \nu\left(p_{1}\right), \ldots, \nu\left(p_{m}\right)\right)$, or even $\left(A, f_{1}, \ldots, f_{n}\right.$, $\left.p_{1}, \ldots, p_{m}\right)$. Moreover, we will substitute $\sigma=\left(f_{1}, \ldots, f_{n}, p_{1}, \ldots, p_{m}\right)$ for $\sigma=$ $(F, P, \mathfrak{a})$.

We turn now to the $B$-valued interpretation of a first order language. Consider an algebraic $B$-system $\mathfrak{A}:=(A, \nu)$ of signature $\sigma:=\sigma(\mathfrak{A}):=(F, P, \mathfrak{a})$.

Let $\varphi\left(x_{0}, \ldots, x_{n-1}\right)$ be some formula of signature $\sigma$ with $n$ free variables. Assume given $a_{0}, \ldots, a_{n-1} \in A$. In this event we may define the Boolean truth value

$$
|\varphi|^{\mathfrak{A}}\left(a_{0}, \ldots, a_{n-1}\right) \in B
$$

of a formula $\varphi$ in $\mathfrak{A}$ for the given values $a_{0}, \ldots, a_{n-1}$ of the variables $x_{0}, \ldots, x_{n-1}$. The definition proceeds by usual recursion on the length of $\varphi$. Considering the 
logical connectives and quantifiers, put

$$
\begin{aligned}
|\varphi \wedge \psi|^{\mathfrak{A}}\left(a_{0}, \ldots, a_{n-1}\right) & :=|\varphi|^{\mathfrak{A}}\left(a_{0}, \ldots, a_{n-1}\right) \wedge|\psi|^{\mathfrak{A}}\left(a_{0}, \ldots, a_{n-1}\right) ; \\
|\varphi \vee \psi|^{\mathfrak{A}}\left(a_{0}, \ldots, a_{n-1}\right) & :=|\varphi|^{\mathfrak{A}}\left(a_{0}, \ldots, a_{n-1}\right) \vee|\psi|^{\mathfrak{A}}\left(a_{0}, \ldots, a_{n-1}\right) ; \\
|\neg \varphi|^{\mathfrak{A}}\left(a_{0}, \ldots, a_{n-1}\right) & :=\neg|\varphi|^{\mathfrak{A}}\left(a_{0}, \ldots, a_{n-1}\right) ; \\
\left|\left(\forall x_{0}\right) \varphi\right|^{\mathfrak{A}}\left(a_{1}, \ldots, a_{n-1}\right) & :=\bigwedge_{a_{0} \in A}|\varphi|^{\mathfrak{A}}\left(a_{0}, \ldots, a_{n-1}\right) ; \\
\left|\left(\exists x_{0}\right) \varphi\right|^{\mathfrak{A}}\left(a_{1}, \ldots, a_{n-1}\right) & :=\bigvee_{a_{0} \in A}|\varphi|^{\mathfrak{A}}\left(a_{0}, \ldots, a_{n-1}\right) .
\end{aligned}
$$

We turn now to the atomic formulas. Suppose that $p \in P$ stands for an $m$-ary predicate, $q \in P$ is a 0 -ary predicate, and $t_{0}, \ldots, t_{m-1}$ are some terms of signature $\sigma$ that take the values $b_{0}, \ldots, b_{m-1}$ when the variables $x_{0}, \ldots, x_{n-1}$ assume the values $a_{0}, \ldots, a_{n-1}$. We proceed with letting

$$
\begin{aligned}
|\varphi|^{\mathfrak{A}}\left(a_{0}, \ldots, a_{n-1}\right):=\nu(q), & \text { if } \varphi=q^{\nu} \\
|\varphi|^{\mathfrak{A}}\left(a_{0}, \ldots, a_{n-1}\right):=\neg d\left(b_{0}, b_{1}\right), & \text { if } \varphi=\left(t_{0}=t_{1}\right) ; \\
|\varphi|^{\mathfrak{A}}\left(a_{0}, \ldots, a_{n-1}\right):=p^{\nu}\left(b_{0}, \ldots, b_{m-1}\right), \quad & \text { if } \varphi=p^{\nu}\left(t_{0}, \ldots, t_{m-1}\right),
\end{aligned}
$$

where $d$ is the $B$-metric on $A$.

A formula $\varphi\left(x_{0}, \ldots, x_{n-1}\right)$ is satisfied in an algebraic $B$-system $\mathfrak{A}$ by the assignment $a_{0}, \ldots, a_{n-1} \in A$ of $x_{0}, \ldots, x_{n-1}$, in symbols $\mathfrak{A} \models \varphi\left(a_{0}, \ldots, a_{n-1}\right)$, provided that $|\varphi|^{\mathfrak{A}}\left(a_{0}, \ldots, a_{n-1}\right)=\mathbb{1}_{B}$. The concurrent expressions are as follows: $a_{0}, \ldots, a_{n-1} \in A$ satisfy $\varphi\left(x_{0}, \ldots, x_{n-1}\right)$ or $\varphi\left(a_{0}, \ldots, a_{n-1}\right)$ holds in $\mathfrak{A}$. In case of the two-point Boolean algebra, we come to the conventional definition of satisfaction in an algebraic system.

Recall that a closed formula $\varphi$ of signature $\sigma$ is a tautology or logically valid formula provided that $\varphi$ holds in every algebraic 2-system of signature $\sigma$.

(1) Consider some algebraic $B$-systems $\mathfrak{A}:=(A, \nu)$ and $\mathfrak{D}:=(D, \mu)$ of the same signature $\sigma$. A mapping $h: A \rightarrow D$ is a homomorphism from $\mathfrak{A}$ to $\mathfrak{D}$ provided that the following hold for all $a_{0}, \ldots, a_{n-1} \in A$ :

(a) $d_{B}\left(h\left(a_{1}\right), h\left(a_{2}\right)\right) \leq d_{A}\left(a_{1}, a_{2}\right)$;

(b) $h\left(f^{\nu}\right)=f^{\mu}$ if $\mathfrak{a}(f)=0$;

(c) $h\left(f^{\nu}\left(a_{0}, \ldots, a_{n-1}\right)\right)=f^{\mu}\left(h\left(a_{0}\right), \ldots, h\left(a_{n-1}\right)\right)$ if $0 \neq n:=\mathfrak{a}(f)$;

(d) $p^{\nu}\left(a_{0}, \ldots, a_{n-1}\right) \leq p^{\mu}\left(h\left(a_{0}\right), \ldots, h\left(a_{n-1}\right)\right)$ where $n:=\mathfrak{a}(p)$.

(2) A homomorphism $h$ is strong provided that $\mathfrak{a}(p):=n \neq 0$ for $p \in P$ and

$$
\geq \bigvee_{a_{0}, \ldots, a_{n-1} \in A} p^{\nu}\left(a_{0}, \ldots, a_{n-1}\right) \wedge d_{D}\left(d_{0}, h\left(a_{0}\right)\right) \wedge \cdots \wedge d_{D}\left(d_{n-1}, h\left(a_{n-1}\right)\right)
$$

for all $d_{0}, \ldots, d_{n-1} \in D$,

If a homomorphism $h$ is injective while (a) and (d) turn into equalities then $h$ is an isomorphism from $\mathfrak{A}$ to $\mathfrak{D}$. Obviously, each surjective isomorphism (in particular, the identity mapping $\operatorname{Id}_{A}: A \rightarrow A$ ) is a strong homomorphism. The composite of (strong) homomorphisms is a (strong) homomorphism as well. Clearly, if $h$ is a homomorphism and $h^{-1}$ is a homomorphism too, then $h$ is an isomorphism. We mention again that in the case of the two-point Boolean algebra we come to the conventional concepts of homomorphism, strong homomorphism, and isomorphism. 
5.3. Descending 2. Before defining the descent of a general algebraic system, consider the descent of the two-point Boolean algebra. Take two arbitrary members $0,1 \in \mathbb{V}^{(B)}$ satisfying the condition $\llbracket 0 \neq 1 \rrbracket=\mathbb{1}_{B}$. For instance, put $0:=\mathbb{O}_{B}^{\wedge}$ and $1:=\mathbb{1}_{B}$. The descent $D$ of the two-point Boolean algebra $\{0,1\}^{B}$ inside $\mathbb{V}^{(B)}$ is a complete Boolean algebra isomorphic to $B$. The formulas $\llbracket \chi(b)=1 \rrbracket=b$ and $\llbracket \chi(b)=0 \rrbracket=\neg b$, with $b \in B$, yield the isomorphism $\chi: B \rightarrow D$.

5.4. Descending an algebraic $\boldsymbol{B}$-system. Look now at an algebraic system $\mathfrak{A}$ of signature $\sigma^{\wedge}$ inside $\mathbb{V}^{(B)}$ and assume that $\mathbb{V}^{(B)} \models$ " $\mathfrak{A}=(A, \nu)$ for some $A$ and $\nu$." The descent of $\mathfrak{A}$ is the pair $\mathfrak{A} \downarrow:=(A \downarrow, \mu)$, where $\mu$ is the function defined by the following formulas:

$$
\begin{gathered}
\mu: f \mapsto(\nu \downarrow(f)) \downarrow \quad(f \in F) ; \\
\mu: p \mapsto \chi^{-1} \circ(\nu \downarrow(p)) \downarrow \quad(p \in P) .
\end{gathered}
$$

Here $\chi$ is the isomorphism of 5.3 between the Boolean algebras $B$ and $\{0,1\}^{B} \downarrow$.

In more detail, the modified descent $\nu \downarrow$ is a mapping with domain $\operatorname{dom}(\nu \downarrow)=$ $F \cup P$. Given $p \in P$, note that $\llbracket \mathfrak{A}(p)^{\wedge}=\mathfrak{A}^{\wedge}\left(p^{\wedge}\right) \rrbracket=\mathbb{1}, \llbracket \nu \amalg(p)=\nu\left(p^{\wedge}\right) \rrbracket=\mathbb{1}$, and so

$$
\mathbb{V}^{(B)} \models \nu \downarrow(p): A^{\mathfrak{a}(f)^{\wedge}} \rightarrow\{0,1\}^{B} .
$$

It is obvious now that $(\nu \downarrow(p)) \downarrow:(A \downarrow)^{\mathfrak{a}(f)} \rightarrow D:=\{0,1\}^{B} \downarrow$ and we may put $\mu(p):=\chi^{-1} \circ(\nu \downarrow(p)) \downarrow$.

Let $\varphi\left(x_{0}, \ldots, x_{n-1}\right)$ be a distinguished formula of signature $\sigma$ with $n$ free variables. Write down the formula $\Phi\left(x_{0}, \ldots, x_{n-1}, \mathfrak{A}\right)$ of the language of set theory which expresses the fact $\mathfrak{A} \models \varphi\left(x_{0}, \ldots, x_{n-1}\right)$. The formula $\mathfrak{A} \models \varphi\left(x_{0}, \ldots, x_{n-1}\right)$ determines an $n$-ary predicate on $A$ or, in other words, a mapping from $A^{n}$ to $\{0,1\}$. By the maximum and transfer principles there is a unique member $|\varphi|^{\mathfrak{A}}$ of $\mathbb{V}^{(B)}$ such that

$$
\begin{gathered}
\llbracket|\varphi|^{\mathfrak{A}}: A^{n^{\wedge}} \rightarrow\{0,1\}^{B} \rrbracket=\mathbb{1}, \\
\llbracket|\varphi|^{\mathfrak{A}}(a \uparrow)=1 \rrbracket=\llbracket \Phi(a(0), \ldots, a(n-1), \mathfrak{A}) \rrbracket=\mathbb{1}
\end{gathered}
$$

for all $a: n \rightarrow A \downarrow$. Instead of $|\varphi|^{\mathfrak{A}}(a \uparrow)$ we will write $|\varphi|^{\mathfrak{A}}\left(a_{0}, \ldots, a_{n-1}\right)$, where $a_{l}:=a(l)$. Therefore, the formula $\mathbb{V}^{(B)} \models " \varphi\left(a_{0}, \ldots, a_{n-1}\right)$ holds in $\mathfrak{A}$ " is valid if and only if $\llbracket \Phi\left(a_{0}, \ldots, a_{n-1}, \mathfrak{A}\right) \rrbracket=\mathbb{1}$.

(2) Let $\mathfrak{A}$ be an algebraic system of signature $\sigma^{\wedge}$ inside $\mathbb{V}^{(B)}$. Then $\mathfrak{A} \downarrow$ is a universally complete algebraic $B$-system of signature $\sigma$. Moreover,

$$
\chi \circ|\varphi|^{\mathfrak{A} \downarrow}=|\varphi|^{\mathfrak{A}} \downarrow
$$

for every formula $\varphi$ of signature $\sigma$.

(3) Let $\mathfrak{A}$ and $\mathfrak{B}$ be two algebraic systems of the same signature $\sigma^{\wedge}$ inside $\mathbb{V}^{(B)}$. Put $\mathfrak{A}^{\prime}:=\mathfrak{A} \downarrow$ and $\mathfrak{B}^{\prime}:=\mathfrak{B} \downarrow$. Then if $h$ is a homomorphism (strong homomorphism) inside $\mathbb{V}^{(B)}$ from $\mathfrak{A}$ to $\mathfrak{B}$ then $h^{\prime}:=h \downarrow$ is a homomorphism (respectively, strong homomorphism) between the $B$-systems $\mathfrak{A}^{\prime}$ and $\mathfrak{B}^{\prime}$.

Conversely, if $h^{\prime}: \mathfrak{A}^{\prime} \rightarrow \mathfrak{B}^{\prime}$ is a homomorphism (strong homomorphism) of the relevant algebraic $B$-systems then $h:=h^{\prime} \uparrow$ is a homomorphism (respectively, strong homomorphism) from $\mathfrak{A}$ to $\mathfrak{B}$ inside $\mathbb{V}^{(B)}$.

(4) Let $\mathfrak{A}:=(A, \nu)$ be an algebraic $B$-system of signature $\sigma$. Then there are $\mathscr{A}$ and $\mu \in \mathbb{V}^{(B)}$ such that

(a) $\mathbb{V}^{(B)} \models "(\mathscr{A}, \mu)$ is an algebraic system of signature $\sigma^{\wedge}$;

(b) if $\mathfrak{A}^{\prime}:=\left(A^{\prime}, \nu^{\prime}\right)$ is the descent of $(\mathscr{A}, \mu)$ then $\mathfrak{A}$ is a universally complete algebraic $B$-system of signature $\sigma$; 
(c) there is an isomorphism $\imath$ from $\mathfrak{A}$ to $\mathfrak{A}^{\prime}$ such that $A^{\prime}=\operatorname{mix}(\imath(A))$;

(d) given a formula $\varphi$ of signature $\sigma$ in $n$ free variables, the following holds

$$
\begin{gathered}
|\varphi|^{\mathfrak{A}}\left(a_{0}, \ldots, a_{n-1}\right)=|\varphi|^{\mathfrak{A}^{\prime}}\left(\imath\left(a_{0}\right), \ldots, \imath\left(a_{n-1}\right)\right) \\
=\chi^{-1} \circ\left(|\varphi|^{\mathfrak{A} \sim}\right) \downarrow\left(\imath\left(a_{0}\right), \ldots, \imath\left(a_{n-1}\right)\right)
\end{gathered}
$$

for all $a_{0}, \ldots, a_{n-1} \in A$, where $\chi$ is defined as above.

\section{Boolean Valued Reals}

We are now able to apply the technique of Boolean valued analysis to the algebraic system of the utmost importance for the whole body of mathematics, the field of the reals. Let us recall a few preliminaries of the theory of vector lattices.

6.1. Kantorovich spaces. We start with some definitions. Members $x$ and $y$ of a vector lattice $E$ are disjoint (in symbols, $x \perp y$ ) provided that $|x| \wedge|y|=0$. The band of $E$ is the disjoint complement $M^{\perp}:=\{x \in E \mid(\forall y \in M) x \perp y\}$ of some nonempty subset $M \subset E$.

The inclusion-ordered set $\mathfrak{B}(E)$ of the bands of $E$ is a complete Boolean algebra under the following operations:

$$
L \wedge K=L \cap K, \quad L \vee K=(L \cup K)^{\perp \perp}, \quad \neg L=L^{\perp} \quad(L, K \in \mathfrak{B}(E)) .
$$

The Boolean algebra $\mathfrak{B}(E)$ is often referred to as the base of $E$.

A band projection in $E$ is an idempotent linear operator $\pi: E \rightarrow E$ such that $0 \leq \pi x \leq x$ for all $0 \leq x \in E$. The set $\mathfrak{P}(E)$ of all band projections, ordered by the rule $\pi \leq \rho \Longleftrightarrow \pi \circ \rho=\pi$, is a Boolean algebra with the following operations:

$$
\pi \wedge \rho=\pi \circ \rho, \quad \pi \vee \rho=\pi+\rho-\pi \circ \rho, \quad \neg \pi=I_{E}-\pi \quad(\pi, \rho \in(E)) .
$$

Assume that $u \in E_{+}$and $e \wedge(u-e)=0$ for some $0 \leq e \in E$. Then $e$ is a fragment or component of $u$. The set $\mathfrak{E}(u)$ of all fragments of $u$ with the induced order from $E$ is a Boolean algebra with the same lattice operations as in $E$ and the complement acting by the rule $\neg e:=u-e$.

A vector lattice $E$ is a Kantorovich space or $K$-space provided that $E$ is Dedekind complete; i.e., each upper bounded nonempty subset of $E$ has the least upper bound.

If each family of pairwise disjoint elements of a $K$-space $E$ is bounded then $E$ is universally complete or extended.

(1) Let $E$ be an arbitrary $K$-space. Then the mapping $\pi \mapsto \pi(E)$ is an isomorphism between the Boolean algebras $\mathfrak{P}(E)$ and $\mathfrak{B}(E)$. If $E$ has an order unit $\mathbb{1}$ then the mappings $\pi \mapsto \pi \mathbb{1}$ from $\mathfrak{P}(E)$ to $\mathfrak{E}(E)$ and $e \mapsto\{e\}^{\perp \perp}$ from $\mathfrak{E}(E)$ to $\mathfrak{B}(E)$ are isomorphisms between the corresponding Boolean algebras.

(2) Each universally complete $K$-space $E$ with order unit $\mathbb{1}$ admits a unique multiplication that makes $E$ into a faithful $f$-algebra and $\mathbb{1}$ into the ring unit. Each band projection $\pi \in \mathfrak{P}(E)$ coincides with multiplication by $\pi(\mathbb{1})$ in the resulting $f$-algebra.

6.2. Descending the reals. By the maximum and transfer principles there is a member $\mathscr{R}$ of $\mathbb{V}^{(B)}$ such that $\mathbb{V}^{(B)} \models$ " $\mathscr{R}$ is an ordered field of the reals." Clearly, the field $\mathscr{R}$ is unique up to isomorphism inside $\mathbb{V}^{(B)}$; i.e., if $\mathscr{R}^{\prime}$ is another field of the reals inside $\mathbb{V}^{(B)}$ then $\mathbb{V}^{(B)} \models$ "the fields $\mathscr{R}$ and $\mathscr{R}^{\prime}$ are isomorphic." It is easy that $\mathbb{R}^{\wedge}$ is an Archimedean ordered field inside $\mathbb{V}^{(B)}$. Therefore we may assume that 
$\mathbb{V}^{(B)} \models " \mathbb{R}^{\wedge} \subset \mathscr{R}$ and $\mathscr{R}$ is a (metric) completion of $\mathbb{R}^{\wedge}$." Given the usual unity 1 of $\mathbb{R}$, note that $\mathbb{V}^{(B)} \models " 1:=1^{\wedge}$ is an order unit of $\mathscr{R}$."

Consider the descent $\mathscr{R} \downarrow$ of the algebraic system $\mathscr{R}:=(|\mathscr{R}|,+, \cdot, 0,1, \leq)$.

(1) In accord with the general construction, we make the descent of the carrier of $\mathscr{R}$ into an algebraic system by descending the operations and order on $\mathscr{R}$. In more detail, if we are given $x, y, z \in \mathscr{R} \downarrow$ and $\lambda \in \mathbb{R}$ then we define the addition, multiplication, and order on $\mathscr{R}$ as follows:

(a) $x+y=z \leftrightarrow \llbracket x+y=z \rrbracket=\mathbb{1}$

(b) $x y=z \leftrightarrow \llbracket x y=z \rrbracket=\mathbb{1}$;

(c) $x \leq y \leftrightarrow \llbracket x \leq y \rrbracket=\mathbb{1}$;

(d) $\lambda x=y \leftrightarrow \llbracket \lambda^{\wedge} x=y \rrbracket=\mathbb{1}$.

(2) The algebraic system $\mathscr{R} \downarrow$ is a universally complete $K$-space. Moreover, there is a (canonical) isomorphism $\chi$ of the Boolean algebra $B$ to the base $\mathfrak{B}(\mathscr{R} \downarrow)$ of $\mathscr{R} \downarrow$ such that

$$
\begin{aligned}
& \chi(b) x=\chi(b) y \leftrightarrow b \leq \llbracket x=y \rrbracket, \\
& \chi(b) x \leq \chi(b) y \leftrightarrow b \leq \llbracket x \leq y \rrbracket
\end{aligned}
$$

for all $x, y \in \mathscr{R} \downarrow$ and $b \in B$.

This remarkable result, establishing the immanent connection between Boolean valued analysis and vector-lattice theory, belongs to Gordon [7. The Gordon theorem has demonstrated that each universally complete Kantorovich space provides a new model of the field of the reals, and all these models have the same rights in mathematics. Moreover, each Archimedean vector, in particular, an arbitrary $L_{p}$-space, $p \geq 1$, ascends into a dense sublattice of the reals $\mathbb{R}$ inside an appropriate Boolean valued universe.

Descending the basic scalar fields opens a turnpike to the intensive application of Boolean valued models in functional analysis. The technique of Boolean valued analysis demonstrates its efficiency in studying Banach spaces and algebras as well as lattice-normed spaces and modules. The corresponding results are collected and elaborated in [8, Chs. 10-12].

\section{The Cardinal Shift}

Boolean valued models were invented for research into the foundations of mathematics. Many delicate properties of the objects inside $\mathbb{V}^{(B)}$ depend essentially on the structure of the initial Boolean algebra $B$. The diversity of opportunities together with a great stock of information on particular Boolean algebras ranks Boolean valued models among the most powerful tools of foundational studies.

The working mathematician feels rarely if ever the dependence of his or her research on foundations. Therefore, we allotted the bulk of the above exposition to the constructions and properties of $\mathbb{V}^{(B)}$ in which the particularities of $B$ were completely immaterial. The basics of Boolean valued analysis rest precisely on these constructions and properties. Neglect notwithstanding, foundations underlie the core of mathematics. Boolean valued analysis stems from the brilliant results of Gödel and Cohen who demonstrated to all of us the independence of the continuum hypothesis from the axioms of ZFC. It thus stands to reason to discuss the relevant matters in some detail. 
7.1. Mysteries of the continuum. The concept of continuum belongs to the most important general tools of science. The mathematical views of the continuum relate to the understanding of time and time-dependent processes in physics. It suffices to mention the great Newton and Leibniz who have different perceptions of the continuum.

The smooth and perpetual motion, as well as vision of the nascent and evanescent arguments producing continuous changes in the dependant variables, underlies Newton's outlook, philosophy, and his method of prime and ultimate ratios. The principal difficulty of the views of Newton rests in the impossibility of imagining the immediately preceding moment of time nor the nearest neighbor of a given point of the continuum. As regards Leibniz, he viewed every varying quantity as piecewise constant to within higher order imperceptible infinitesimals. His continuum splits into a collection of disjoint monads, these immortal and mysterious ideal entities.

The views of Newton and Leibniz summarized the ideas that stem from the remote ages. The mathematicians of the ancient Hellada distinguished between points and monads, so explicating the dual nature of the objects of mathematics. The mystery of the structure of the continuum came to us from our ancestors through two millennia.

The set-theoretic stance revealed a new enigma of the continuum. Cantor demonstrated that the set of the naturals is not equipollent with the simplest mathematical continuum, the real axis. This gave an immediate rise to the problem of the continuum which consists in determining the cardinalities of the intermediate sets between the naturals and the reals. The continuum hypothesis reads that the intermediate subsets possess no new cardinalities.

The continuum problem was the first in the already cited report by Hilbert [3]. An incontrovertible anti-ignorabimus, Hilbert was always inclined to the validity of the continuum hypothesis. It is curious that one of his most beautiful and appealing articles [5], which is dated as of 1925 and contains the famous phrase about Cantor's paradise, was devoted in fact to an erroneous proof of the continuum hypothesis.

The Russian prophet Luzin viewed as implausible even the mere suggestion of the independence of the continuum hypothesis. He said in his famous talk "Real Function Theory: State of the Art" 9] at the All-Russia Congress of Mathematicians in 1927: "The first idea that might leap to mind is that the determination of the cardinality of the continuum is a matter of a free axiom like the parallel postulate of geometry. However, when we vary the parallel postulate, keeping intact the rest of the axioms of Euclidean geometry, we in fact change the precise meanings of the words we write or utter, that is, 'point,' 'straight line,' etc. What words are to change their meanings if we attempt at making the cardinality of the continuum movable along the scale of alephs, while constantly proving consistency of this movement? The cardinality of the continuum, if only we imagine the latter as a set of points, is some unique entity that must reside in the scale of alephs at the place which the cardinality of the continuum belongs to; no matter whether the determination of this place is difficult or even 'impossible for us, the human beings' as J. Hadamard might comment."

Gödel proved the consistency of the continuum hypothesis with the axioms of ZFC, by inventing the universe of constructible sets [10. Boolean valued models made Cohen's difficult result simple, demonstrating to the working mathematician the independence of the continuum hypothesis with the same visuality as the 
Poincaré model for non-Euclidean geometry. Those who get acquaintance with this technique are inclined to follow Cohen [2] and view the continuum hypothesis as "obviously false."

We will keep to the famous direction of Dedekind that "whatever is provable must not be believed without proof." So we will briefly discuss the formal side of the matter. More sophisticated arguments are collected in the remarkable articles by Kanamori [1], Cohen [12, and Manin [13.

7.2. Ordinals. The concept of ordinal reflects the ancient trick of counting with successive notches.

A class $X$ is transitive provided that each member of $X$ is a subset of $X$; i.e.,

$$
\operatorname{Tr}(X):=(\forall y)(y \in X \rightarrow y \subset X) .
$$

An ordinal class is a transitive class well ordered by the membership relation $\epsilon$. The record $\operatorname{Ord}(X)$ means that $X$ is an ordinal class. An ordinal class presenting a set is an ordinal or a transfinite number. The class of all ordinals is denoted by On. The small Greek letters usually stand for the ordinals. Moreover, the following abbreviations are in common parlance: $\alpha<\beta:=\alpha \in \beta, \alpha \leq \beta:=(\alpha \in \beta) \vee(\alpha=\beta)$, and $\alpha+1:=\alpha \cup\{\alpha\}$. If $\alpha<\beta$ then $\alpha$ preceeds $\beta$ and $\beta$ succeds $\alpha$.

Appealing to the axiom of foundation, we easily come to a simpler definition: an ordinal is a transitive set totally ordered by membership:

$$
\operatorname{Ord}(X) \leftrightarrow \operatorname{Tr}(X) \wedge(\forall u \in X)(\forall v \in X)(u \in v \vee u=v \vee v \in u)
$$

Note the useful auxiliary facts:

(1) Let $X$ and $Y$ be some classes. If $X$ is an ordinal class, is transitive, and $X \neq Y$ then $Y \subset X$ if and only if $Y \in X$.

(2) The intersection of two ordinal classes is an ordinal class too.

(3) If $X$ and $Y$ are ordinal classes then $X \in Y \vee X=Y \vee Y \in X$.

(4) The following hold:

(a) the members of an ordinal class are ordinals;

(b) On is the only ordinal class other than any ordinal;

(c) if $\alpha$ is an ordinal then $\alpha+1$ is the least ordinal among the successors of $\alpha$;

(d) the union $\bigcup X$ of a nonempty class of ordinals $X \subset$ On is an ordinal class too; if $X$ is a set then $\bigcup X$ is the least upper bound for $X$ in the ordered class On.

(5) The least upper bound of a set of ordinals $x$ is usually denoted by $\lim (x)$. An ordinal $\alpha$ is limit provided that $\alpha \neq \varnothing$ and $\lim (\alpha)=\alpha$. Equivalently, $\alpha$ is a limit ordinal whenever $\alpha$ admits no presentation in the form $\alpha=\beta+1$ with any $\beta \in$ On. The symbol $K_{\text {II }}$ stands for the class of all limit ordinals. The ordinals, residing beyond $K_{\mathrm{II}}$, comprise the class of nonlimit ordinals $K_{\mathrm{I}}:=$ On $\backslash K_{\mathrm{II}}=\{\alpha \in$ On $\mid$ $(\exists \beta \in \mathrm{On})(\alpha=\beta+1)\}$. Denote by $\omega$ the least limit ordinal. It gives pleasure to recall that the members of $\omega$ are positive integers.

(6) Since $\operatorname{Ord}(x)$ is a restricted formula for $x \in \mathbb{V}$, it follows from 4.1 (4) that

$$
\alpha \in \operatorname{On} \leftrightarrow \mathbb{V}^{(B)} \models \operatorname{Ord}\left(\alpha^{\wedge}\right) .
$$

(7) $\mathbb{V}^{(B)} \models \operatorname{Ord}(x)$ holds for $x \in \mathbb{V}^{(B)}$ if and only if there are an ordinal $\beta \in$ On and a partition of unity $\left(b_{\alpha}\right)_{\alpha \in \beta} \subset B$ such that $x=\operatorname{mix}_{\alpha \in \beta}\left(b_{\alpha} \alpha^{\wedge}\right)$. 
7.3. Cardinals. The concept of cardinal stems from the palaeolithic trick of counting by comparison with an agreed assemblage. This trick is witnessed by many archeological bullas with tokens.

Two sets are equipollent or have the same cardinality provided that there is a bijection from one of them onto the other. An ordinal not equipollent to any of its predecessors is a cardinal. In other words, the cardinals make the scale of standards for comparing cardinalities and serve as referents of the measure of quantity. Every positive integer is a cardinal. Clearly, $\omega$ is the least infinite cardinal. To each infinite ordinal $\alpha$ there is a unique least cardinal $\mathscr{H}(\alpha)$ greater than $\alpha$. These resulting cardinals are usually called alephs and it is customary to use in their symbolizing the traditional and somewhat excessive notations:

$$
\begin{gathered}
\aleph_{0}:=\omega_{0}:=\omega ; \\
\aleph_{\alpha+1}:=\omega_{\alpha+1}:=\mathscr{H}\left(\omega_{\alpha}\right) \quad\left(\alpha \in K_{\mathrm{I}}\right) ; \\
\aleph_{\beta}:=\omega_{\beta}:=\lim \left\{\omega_{\alpha} \mid \alpha \in \beta\right\} \quad\left(\beta \in K_{\mathrm{II}}\right) .
\end{gathered}
$$

(1) Each set $x$ is equipollent with the unique cardinal $|x|$ called the cardinal number or cardinality of $x$. A set $x$ is countable in case $|x|=\omega_{0}:=\omega$ and at most countable, in case $|x| \leq \omega_{0}$.

(2) The standard names of ordinals and cardinals are standard ordinals and standard cardinals. By the transfer principle, to each standard ordinal that serves as the name of $\alpha \in$ On, inside $\mathbb{V}^{(B)}$ there corresponds the unique aleph $\aleph_{\alpha^{\wedge}}$ since $\llbracket \operatorname{Ord}\left(\alpha^{\wedge}\right) \rrbracket=\mathbb{1}$. As mentioned in 7.2 (7), an arbitrary ordinal inside $\mathbb{V}^{(B)}$ is a mixing of standard ordinals. Similarly, each Boolean valued cardinal is a mixing of some family of standard cardinals.

(3) $\llbracket\left(\omega_{\alpha}\right)^{\wedge} \leq \aleph_{\alpha^{\wedge}} \rrbracket=\mathbb{1}$ for every cardinal $\alpha$.

(4) A Boolean algebra $B$ enjoys the countable chain condition provided that every disjoint subset of $B$ is at most countable. In such an algebra we have $\operatorname{Card}(\alpha) \rightarrow \mathbb{V}^{(B)} \models\left(\omega_{\alpha}\right)^{\wedge} \leq \aleph_{\alpha^{\wedge}}$ and, moreover, $\mathbb{V}^{(B)} \models\left(\omega_{\alpha}\right)^{\wedge}=\aleph_{\alpha^{\wedge}}$ for all $\alpha \in$ On.

7.4. The continuum hypothesis. Given an ordinal $\alpha$, denote by $2^{\omega_{\alpha}}$ the cardinality of the powerset $\mathscr{P}\left(\omega_{\alpha}\right)$; i.e., $2^{\omega_{\alpha}}:=\left|\mathscr{P}\left(\omega_{\alpha}\right)\right|$. This notation is justified since $2^{x}$ and $\mathscr{P}(x)$ are equipollent for all $x$, where $2^{x}$ is the class of all mappings from $x$ to 2. Cantor discovered and demonstrated that $|x|<\left|2^{x}\right|$ for every set $x$. In particular, $\omega_{\alpha}<2^{\omega_{\alpha}}$ for each ordinal $\alpha$. By definition, we see that $\omega_{\alpha+1} \leq 2^{\omega_{\alpha}}$.

The question of whether or not there are some intermediate cardinalities between $\omega_{\alpha+1}$ and $2^{\omega_{\alpha}}$; i.e., whether or not $\omega_{\alpha+1}=2^{\omega_{\alpha}}$, is the content of the generalized problem of the continuum. In case $\alpha=0$, this is the classical problem of the continuum.

The continuum hypothesis is in common parlance the equality $\omega_{1}=2^{\omega}$. The continuum hypothesis enables us to well order every segment of the straight line so that every subsegment with respect to the new order will be at most countable. The absence of intermediate cardinalities, expressed as $(\forall \alpha \in \mathrm{On}) \omega_{\alpha+1}=2^{\omega_{\alpha}}$, is the generalized continuum hypothesis.

7.5. Algebras of forcing. We turn now to describing some special class of Boolean algebras $B$ that opens up many amazing possibilities such as "gluing together" two standard infinite cardinals as well as various shifts along the alephic scale inside an appropriate Boolean valued universe $\mathbb{V}^{(B)}$. 
(1) Consider an ordered set $P:=(P, \leq)$. Assume that $P$ has the least element $\mathbb{0}$. This involves no loss in generality since in case $P$ lacks the bottom, we can always supply the latter by replacing $P$ with $P \cup\{0\}$. We define the binary relation $\perp$ on $P$ by letting

$$
p \perp q \leftrightarrow(\forall r \in P)(r \leq p \wedge r \leq q \rightarrow r=\mathbb{0}) .
$$

Consider the polar $A^{\perp}:=\pi_{\perp}(A):=\{q \mid(\forall p \in A) q \perp p\}$ and use the abbreviation $[p]:=\{p\}^{\perp \perp}$. The relation $\perp$ is symmetric. Moreover, if $p \perp p$ then $p=\mathbb{O}$. In particular, the least $\perp$-band $P^{\perp}$ coincides with $\{\mathbb{O}\}$. It is easy to check that $p \mapsto[p]$ is a monotonic mapping and $\perp$ is a disjointness on $P$. By the general properties of disjointness, the inclusion ordered set $\mathfrak{K}_{\perp}(P)$ of all $\perp$-bands in $P$ is a complete Boolean algebra. Recall that a subset $P$ of $B$ is dense provided that to each nonzero $b \in B$ there is a nonzero $p \in P$ satisfying $p \leq b$.

(2) For an ordered set $P$ the following are equivalent:

(a) if $p, q \in P$ and $\mathbb{O} \neq q \nless p$ then there is a nonzero $p^{\prime} \in P$ satisfying $p^{\prime} \leq q$ and $p \perp p^{\prime}$;

(b) $[p]=[\mathbb{0}, p]$ for all $p \in P$;

(c) $p \mapsto[p]$ is one-to-one;

(d) $p \mapsto[p]$ is an order isomorphism of $P$ onto a dense subset of the complete Boolean algebra $\mathfrak{K}_{\perp}(P)$.

An ordered set $P$ is refined provided that $P$ enjoys one (and, hence, all) of the conditions (a)-(d) of 7.5 (2). In other words, the refined ordered sets are, up to isomorphism, the dense subsets of complete Boolean algebras. The complete Boolean algebra $\mathfrak{K}_{\perp}(P)$ is usually referred to as the Boolean completion of $P$. We proceed now to the examples of refined ordered sets of use in the sequel.

(3) Take two nonempty sets $x$ and $y$. Denote by $C(x, y)$ the set of all functions with domain a finite subset of $x$ and range in $y$. In other words,

$$
C(x, y):=\left\{f \mid \operatorname{Fnc}(f) \wedge \operatorname{dom}(f) \in \mathscr{P}_{\text {Fin }}(x) \wedge \operatorname{im}(f) \subset y\right\} .
$$

Equip $C(x, y)$ with the following order $g \leq f \leftrightarrow g \supset f$. If $g \nless f$ then $\operatorname{dom}(f) \subset$ $\operatorname{dom}(g)$ and $f \neq\left. g\right|_{\operatorname{dom}(f)}$ or, otherwise, $\operatorname{dom}(f)$ does not lie in $\operatorname{dom}(g)$.

In the first case, put $f^{\prime}:=g$; and, in the second case, define $f^{\prime}$ by the rules $\operatorname{dom}\left(f^{\prime}\right):=\operatorname{dom}(f) \cup \operatorname{dom}(g),\left.f^{\prime}\right|_{\operatorname{dom}(g)}=g$, and $\left.f^{\prime}\right|_{z} \neq\left. f\right|_{z}$, where $z:=\operatorname{dom}(f) \backslash$ $\operatorname{dom}(g)$. In both cases it is easy that $f^{\prime} \leq f$ and $f \perp f^{\prime}$. Hence, $C(x, y) \cup\{\mathbb{O}\}$ is a refined ordered set. We denote the Boolean completion of $C(x, y)$ by $B(x, y)$.

(4) Let $\varkappa$ be an infinite cardinal. Assume that $x$ and $y$ are the same as in (3), while $|y| \geq 2$. Denote by $C_{\varkappa}(x, y)$ the set of all functions whose domain lies in $x$ and has cardinality strictly less than $\varkappa$. In other words,

$$
C_{\varkappa}(x, y):=\{f|\operatorname{Fnc}(f) \wedge \operatorname{dom}(f) \in \mathscr{P}(x) \wedge| \operatorname{dom}(f) \mid<\varkappa \wedge \operatorname{im}(f) \subset y\} .
$$

Order $C_{\varkappa}(x, y)$ in the same manner as in (3). Arguing as above, we see that $C_{\varkappa}(x, y) \cup\{\mathbb{0}\}$ is a refined ordered set. We denote the Boolean completion of $C_{\varkappa}(x, y)$ by $B_{\varkappa}(x, y)$. Clearly, $C(x, y)=C_{\omega}(x, y)$ and $B(x, y)=B_{\omega}(x, y)$.

7.6. Shifting alephs. The collection of Boolean algebras in 7.5 enables us to achieve a cardinal shift in an appropriate Boolean valued model, so demonstrating Cohen's beautiful result. In his talk of 2001 on the discovery of forcing, Cohen mentioned that "using the language of Boolean algebras brings our technique of forcing to standard usages" [12, p. 1096]. 
(1) Let $\lambda$ be an arbitrary infinite cardinal. Consider the complete Boolean algebra $B(\omega, \lambda)$. Then $\left|\lambda^{\wedge}\right|$ is the countable cardinal inside $\mathbb{V}^{(B)}$; i.e., $\mathbb{V}^{(B)} \models\left|\lambda^{\wedge}\right|=$ $\aleph_{0}$

(2) Given two infinite cardinals $\varkappa$ and $\lambda$, there is a complete Boolean algebra $B$ such that

$$
\mathbb{V}^{(B)} \models\left|\varkappa^{\wedge}\right|=\left|\lambda^{\wedge}\right|
$$

(3) $B(x, 2)$ is a Boolean algebra with the countable chain condition.

(4) Let $x$ be a nonempty set and $|x|=\omega_{\alpha}$. Then

$$
\omega_{\alpha} \leq|B(x, 2)| \leq\left(\omega_{\alpha}\right)^{\omega_{0}} .
$$

(5) If $\left(\omega_{\alpha}\right)^{\omega_{0}}=\omega_{\alpha}$ and $B:=B\left(\omega \times \omega_{\alpha}, 2\right)$ then $\mathbb{V}^{(B)} \models 2^{\aleph_{0}}=\aleph_{\alpha^{\wedge}}$.

(6) If $\mathrm{ZF}$ is consistent then $\mathrm{ZFC}$ remains consistent together with the axiom $2^{\omega_{0}}=\omega_{2}$.

Indeed, Gödel demonstrated [10] that ZF remains consistent on assuming the generalized continuum hypothesis. Hence,

$$
\left(\omega_{2}\right)^{\omega_{0}}=\left(2^{\omega_{1}}\right)^{\omega_{0}}=2^{\omega_{1} \cdot \omega_{0}}=2^{\omega_{1}}=\omega_{2} .
$$

By (5) there is a complete Boolean algebra $B$ such that $\mathbb{V}^{(B)} \models 2^{\aleph_{0}}=\aleph_{2^{\wedge}}$. Since $\mathbb{V}^{(B)} \models 2^{\wedge}=2$, it is obvious that $\mathbb{V}^{(B)} \models \aleph_{2^{\wedge}}=\aleph_{2}$ and so $\mathbb{V}^{(B)} \models 2^{\aleph_{0}}=\aleph_{2}$. Moreover, $\mathbb{V}^{(B)} \models$ ZFC by the transfer principle $3.2(1)$.

(7) Continuity of all homomorphisms of the classical Banach algebra $C([0,1])$ is independent of the rest of the axioms of ZFC but depends on the cardinality of $2^{\omega_{0}}$. On this matter, see [14 p. 19].

(8) Let $A$ stand for the set of functions from $\mathbb{R}$ to countable subsets of $\mathbb{R}$. If $f \in A$ while $x$ and $y$ are some random reals then it is reasonable to believe that $y \in f(x)$ with probability 0 ; i.e., $x \notin f(y)$ with probability 1 . Similarly, $y \notin f(x)$ with probability 1. Using these instances of the common sense, Freiling proposed [15] the axiom $\mathrm{AX}$ which reads

$$
(\forall f \in A)(\exists x)(\exists y) x \notin f(y) \wedge y \notin f(x) .
$$

It turns out that AX amounts to the negation of the continuum hypothesis, $\mathrm{CH}$, in ZFC. This is another argument in favor of the Cohen statement: "I think the consensus will be that $\mathrm{CH}$ is false" [12, p. 1099].

The above approach to the problem of the continuum proceeds mainly along the lines of [16. As regards the omitted details and references, see [8, Ch. 9]. The state of the art in forcing is presented in [17.

\section{Logic and Freedom}

Mathematics is the most ancient of sciences. However, in the beginning was the word. We must remember that the olden "logos" resides in logics and logistics rather than grammar. The order of mind and the order of store are the precious gifts of our ancestors.

The intellectual field resides beyond the grips of the law of diminishing returns. The more we know, the huger become the frontiers with the unbeknown, the oftener we meet the untangled. The twentieth century enriched our geometrical views with the concepts of space-time and fractality. Each instance of knowledge is an event, a point in the Minkowski 4-space. The realm of our knowledge comprises a clearly bounded set of these instances. The frontiers of science produce the boundary between the known and the unknown which is undoubtedly fractal and we have no 
grounds to assume it rectifiable or measurable. It is worth noting in parentheses that rather smooth are the routes to the frontiers of science which are charted by teachers, professors, and all other kinds of educationalists. Pedagogics dislikes saltations and sharp changes of the prevailing paradigm. Possibly, these topological obstructions reflect some objective difficulties in modernizing education.

The proofs are uncountable of the fractality of the boundary between the known and the unbeknown. Among them we see such negative trends as the unleashed growth of pseudoscience, mysticism, and other forms of obscurantism which creep into all lacunas of the unbeknown. As revelations of fractality appear the most unexpected, beautiful, and stunning interrelations between seemingly distant areas and directions of science.

The revolutionary changes in mathematics in the vicinity of the turn of the twentieth century are connected not only with the new calculus of the infinite which was created by Cantor. Of similar import was the rise and development of mathematical logic which applied rigor and analysis to the very process of mathematical demonstration. The decidable and the undecidable, the provable and the improvable, the consistent and the inconsistent have entered the research lexicon of the perfect mathematician. Mathematics became a reflexive science that is engaged not only in search of truths but also in study of its own methods for attaining these truths.

Aristotle's logic, the paradoxes of Zeno, the razor of William of Occam, the donkey of Buridan, the Lebnizian Calculemus, and Boolean algebras are the outstanding achievements of the mankind which cast light on the road to the new stages of logical studies. Frege immortalized his name by inventing the calculus of predicates which underlies the modern logic.

The twentieth century is marked with deep penetration of the ideas of mathematical logic into many sections of science and technology. Logic is a tool that not only organizes and orders our ways of thinking but also liberates us from dogmatism in choosing the objects and methods of research. Logic of today is the best instrument and institution of mathematical freedom. Boolean valued analysis serves as a brilliant confirmation of this thesis.

Returning to Takeuti's original definition of Boolean valued analysis, we must acknowledge its extraordinary breadth. The Boolean valued model resting on the dilemma of "verum" and "falsum" is employed implicitly by the overwhelming majority of mathematicians. Our routine talks and discussions on seminars hardly deserve the qualification of articles of prose. By analogy, it seems pretentious to claim that Euler, Cauchy, and Abel exercised Boolean valued analysis.

Boolean valued analysis is a special mathematical technique based on validating truth by means of a nontrivial Boolean algebra. From a category-theoretic viewpoint, Boolean valued analysis is the theory of Boolean toposes. From a topological viewpoint, it is the theory of continuous polyverses over Stone spaces.

Mach taught us the economy of thought. It seems reasonable to apply his principle and to shorten the bulky term "Boolean valued analysis." Mathematization of the laws of thought originated with Boole [18 and deserves the lapidary title "Boolean analysis." 


\section{REFERENCES}

[1] Takeuti G. (1978) Two Applications of Logic to Mathematics. Tokio-Princeton: Iwanami Publ. \& Princeton University Press.

[2] Cohen P. J.(1966) Set Theory and the Continuum Hypothesis. New York etc.: Benjamin.

[3] Hilbert D. (1902) "Mathematical problems. Lecture delivered before the International Congress of Mathematicians at Paris in 1900," Bull. Amer. Math. Soc., 8, 437-479.

[4] Johnstone P. T. (2002) Sketches of an Elephant. A Topos Theory Compendium. Oxford: Clarendon Press (Oxford Logic Guides; 438).

[5] Hilbert D. (1967) "On the infinite," In: From Frege to Gödel 1879-1931: A Source Book in the History of Science. Cambridge: Harvard University Press, 367-392.

[6] Hofstedter D. R. (1999) Gödel, Escher, Bach: an Eternal Golden Braid (20th Anniversary Edition). New York: Basic Books.

[7] Gordon E. I. (1977) "Real numbers in Boolean-valued models of set theory, and $K$-spaces," Soviet Math. Doklady, 18, pp. 1481-1484.

[8] Kusraev A. G. and Kutateladze S. S. (2005) Introduction to Boolean Valued Analysis. Moscow: Nauka Publishers (in Russian).

[9] Luzin N. N. (1928) "Real function theory: the state of the art," In: Proceedings of the All-Russia Congress of Mathematicians (Moscow, April 27-May 4, 1927), Moscow and Leningrad: Glavnauka, 11-32.

[10] Gödel K. (1940) The Consistency of the Axiom of Choice and of the Generalized Continuum Hypothesis. Princeton: Princeton Univ. Press.

[11] Kanamori A. (1996) "The mathematical developments of set theory from Cantor to Cohen," Bull. Symbolic Logic, 1:1, 1-70.

[12] Cohen P. (2002) "The discovery of forcing," Rocky Mountain J. Math., 32:4, 1071-1100.

[13] Manin Yu. I. (2004) "Georg Cantor and his heritage," Proceedings of the Steklov Institute, 246, 208-216.

[14] Dales Y. G. and Oliveri G. (Eds.) (1998) Truth in Mathematics. Oxford: Clarendon Press.

[15] Freiling Ch. (1986) "Axioms of symmetry: throwing darts at the real line," / J. Symbolic Logic, 51, 190-200.

[16] Bell J. L. (2005) Set Theory: Boolean-Valued Models and Independence Proofs. Oxford: Clarendon Press (Oxford Logic Guides; 47).

[17] Shelah S. (1998) Proper and Improper Forcing. Berlin: Springer-Verlag.

[18] Boole G. (1997) Selected Manuscripts on Logic and Its Philosophy. Basel: Birkhäuser-Verlag (Science Networks. Historical Studies; 20).

Sobolev Institute of Mathematics

Novosibirsk 630090

Russia

E-mail address: $\quad$ sskut@member.academ.org 